\title{
La etnobotánica moqoit inédita de Raúl Martínez Crovetto I: DESCRIPCIÓN, ACTUALIZACIÓN Y ANÁLISIS DE LA NOMENCLATURA INDÍGENA
}

\author{
GUSTAVO F. SCARPA ${ }^{1}$ y CINTIA N. ROSSO ${ }^{2}$
}

\begin{abstract}
Summary: The unpublished moqoit ethnobotany of Martínez Crovetto I: Description, updating and analysis of the indigenous nomenclature. Raúl Martínez Crovetto (M.C.) is the begginer of the ethnobotanical researches in Argentina. Valuable and unpublished data from this author about mocoví (moqoit) indians ethnobotany recorded in the '60's in the Chaco province, were found at the Instituto de Botánica del Nordeste. The objetive of this paper is to rescue, to analyze and to interpret the huge volume of information about mocovi plant names recorded by M.C. The aim is to contribute to the cultural patrimony of these people and to the understanding of their ethnobotany. Methodology employed is the corresponding to the "historical ethnobotany", that considers past records as primary sources, about which classical ethnobotanical method is applied. M.C. records 295 plant names corresponding to 443 botanical taxa, most of them native species (95\%). Semantic analysis of these names resulted in 84 primary lexemes (proper names); 37 secondary lexemes, and 141 metaphorical-descriptive names, besides some translation and absolute loanwords taken from other languages. Edition and analysis of the substantial information here presented -a third of which is updated with accuracy-and the ethnotaxonomy implications it shows, is highlighted.
\end{abstract}

Key words: Ethnobotany, mocoví, Chaco, Martínez Crovetto, vernacular names.

Resumen: Raúl Martínez Crovetto (M.C.) es considerado pionero de las investigaciones etnobotánicas argentinas. En el Instituto de Botánica del Nordeste se hallaron valiosos manuscritos inéditos de este autor sobre la etnobotánica mocoví (moqoit) documentados en la década de 1960 en la provincia del Chaco. El objetivo de este trabajo es rescatar, analizar e interpretar el voluminoso conjunto de informaciones que M.C. ha relevado sobre la fitonimia mocoví, a los fines de contribuir al patrimonio cultural de este pueblo y a la comprensión de su etnobotánica. La metodología empleada es la correspondiente a la "etnobotánica histórica" que considera a los datos del pasado como una fuente de información primaria, sobre los cuales se aplica el método etnobotánico clásico. Se comprueba que M.C. registra un total de 295 fitónimos moqoit correspondientes a 443 entidades botánicas totales, la mayoría nativas (95\%). El análisis semántico de la nomenclatura vernácula consigna 84 lexemas primarios, 37 lexemas secundarios y 141 nombres metafórico-descriptivos, además de algunos préstamos de traducción y absolutos re-fonologizados tomados de otras lenguas. Se destacan la edición y el análisis de la cuantiosa información aquí referida, de la cual un tercio es aquí actualizada y precisada, así como sus implicancias etnotaxonómicas.

Palabras clave: Etnobotánica, mocoví, Chaco, Martínez Crovetto, nombres vernáculos.

\footnotetext{
1 Investigador Adjunto del CONICET. Museo Argentino de Ciencias Naturales "Bernardino Rivadavia", Angel Gallardo 470, C1405DJR Buenos Aires.

2 Becaria posdoctoral del CONICET. Centro de Estudios Farmacológicos y Botánicos. Paraguay 2155 piso 16,1121 Buenos Aires.
} 


\section{INTRODUCCIÓN}

Durante el desarrollo de una investigación etnobotánica en curso sobre la etnia moqoit (mocoví) los autores tuvimos la oportunidad de encontrar en la biblioteca del IBONE (UNNE, Corrientes) una cuantiosa información inédita sobre esta temática, la cual fuera recabada durante 1967 en el sudoeste de la provincia del Chaco por el Dr. Raúl Martínez Crovetto. Entre los antecedentes históricos sobre la fitonimia moqoit, en particular, figuran los del sacerdote jesuita Paucke (19421944) (siglo XVIII) y los de Zapata Gollán (1945) (mediados del XX), además de escasos datos publicados por Martínez Crovetto (1967; 1968a; 1968b; 1968c, 1970). En efecto, a pesar que en estos trabajos M.C. indica las cantidades de fitónimos y de plantas útiles de los moqoit, los únicos nombres indígenas -con su correspondiente identificación botánica- son los referidos a sus plantas cultivadas (25) o aquellos usados para ejemplificar cada una de las categorías tipológicas de su clasificación fitonímica. En la actualidad, a excepción del estudio de Rosso (2010) -sobre un aspecto de su fitonimia recopilada en la actualidad-, el de Scarpa \& Rosso (2011) -que analiza la significación del "coro" (Nicotiana paa Mart. Crov.)- y el de Rosso \& Scarpa (2012) -sobre los datos etnobotánicos en la obra de Paucke-, no han sido publicados otros trabajos acerca de la etnobotánica moqoit. Debido a la escasez de informaciones sobre esta temática, los datos inéditos considerados en este trabajo sobre la fitonimia de este pueblo cobran una relevancia particular, ya que constituirían los registros etnobotánicos más exhaustivos hallados sobre esta etnia hasta la actualidad.

Los mocovíes eran en el pasado un pueblo semi-nómade de cazadores-recolectores, pescadores y horticultores incipientes que históricamente se ubicaron en la región sur del Chaco Oriental argentino. Su modo de vida ha cambiado profundamente de tal manera que en la actualidad se conchaban como jornaleros en trabajo agrícolas o forestales, como docentes u otro trabajo asalariado o bien arriendan o trabajan sus propias fincas. En la actualidad, las comunidades mocovíes se encuentran ubicadas en su gran mayoría en el sur de la provincia de Chaco y el norte de la provincia de Santa Fe. El idioma moqoit - mocoví- hablado por este grupo indígena pertenece a la familia lingüística guaycurú y posee dos variedades dialectales correspondientes a las localizaciones antes mencionadas (Rosso, 2010). Si bien la castellanización es un hecho consumado, el idioma originario mantiene un grado aceptable de vitalidad en gran parte de las comunidades chaqueñas, mientras que en Santa Fe el abandono de la lengua, de su uso y su transmisión, se ha acelerado en la segunda mitad del siglo XX determinando un creciente debilitamiento de la misma (Gualdieri, 2006).

Los estudios sobre la nomenclatura vernácula asignada a las plantas por un pueblo dado poseen una importancia de índole lexicográfica, etnobotánica y etnotaxonómica. Desde el enfoque lingüístico, cada una de las denominaciones a partir de las cuales los pueblos refieren a las plantas constituye una "entrada" léxica de los diccionarios de lenguas indígenas, conjuntamente con sus correspondientes identificaciones botánicas (muchas veces claramente incompletas o imprecisas cuando estas no se realizan sobre la base de estudios etnobotánicos). La clase de nombres empleados por ejemplo, aporta un dato de valor sobre el significado general de la planta a la cual se hace referencia; en efecto, se ha registrado que las principales plantas para la cultura de un pueblo se denominan con lexemas primarios (Scarpa, 2007). Desde el punto de vista etnotaxonómico, por otra parte, algunos de los fitónimos constituyen verdaderas categorías clasificatorias vernáculas (como algunos nombres genéricos), o bien incluyen en sí mismos las marcas de clase a las que se hallan incluidos, lo cual resulta sumamente relevante para el análisis de su etnotaxonomía (ver Scarpa, 2010; Rosso, 2010).

Raúl Martínez Crovetto (M.C.), profesor durante veinticuatro años de la cátedra de Botánica Sistemática y Fitogeografía en la Facultad de Ciencias Agrarias de la UNNE, es considerado el pionero de las investigaciones etnobotánicas propiamente dichas en Argentina (Arenas, 1992). Además de la precisión botánica -y etnográficade estos datos inéditos, el hecho que hayan sido registrados hace ya más de 45 años le confieren una importancia adicional en vistas al cambio cultural sobrevenido en esta etnia (que habría determinado el olvido y/o la pérdida de prácticas y conocimientos), así como a la alta tasa de deforestación sufrida en todo el Chaco Oriental 


\section{G. F. Scarpa y C. N. Rosso - La etnobotánica moqoit inédita de Raúl Martínez Crovetto}

desde esa época a la actualidad ${ }^{3}$. Así, por una u otra razón, muchos de los datos recopilados por M.C. resultan imposibles de volver a registrarse a campo en la actualidad. La validez y relevancia de otros datos inéditos de este autor ya fueron destacadas en otras obras donde algunos de ellos han sido publicados (Viegas Barros, 1995; Pirondo \& Keller, 2012).

En suma, el objetivo de esta contribución es rescatar, analizar, actualizar e interpretar el voluminoso conjunto de informaciones de M.C. acerca de la fitonimia mocoví en particular y de su etnobotánica en general que hasta hoy permanecen inéditas, a los fines de contribuir al patrimonio cultural y a la comprensión de la etnobotánica actual de este grupo humano.

\section{Material y Métodos}

Los materiales cuyo rescate, actualización y análisis se efectúan en este trabajo provienen de manuscritos inéditos de M.C. encontrados en la Biblioteca del Instituto de Botánica del Nordeste (IBONE) de la Facultad de Ciencias Agrarias de la Universidad Nacional del Nordeste (UNNE), sito en la ciudad de Corrientes, entre abril de 2010 y octubre de 2011. Los mismos se hallaron en una caja de cartón rotulada: "Caja Mocoví - Ms. Martínez Crovetto", ordenados en tres paquetes de fichas atadas y etiquetados de la siguiente manera: 1- "Mocobí - latino. 327 nombres (10/1/67)"; 2- "Latino - Mocoví. 475 sp. (9/9/67)" y 3- "Mocovíes. 11/10/67". El primero de ellos lo conformaban 323 fichas inicializadas en su borde superior izquierdo con uno o más fitónimos en lengua moqoit ordenadas alfabéticamente según este criterio. En renglones subsiguientes figuraba el o los nombres científicos a los que correspondían dicho/s nombre/s vernáculo/s. El segundo paquete contaba con 475 fichas inicializadas por un nombre

\footnotetext{
${ }^{3}$ A pesar de que la zona donde el citado investigador registró sus datos es históricamente agrícola -antes con cultivos de algodón preferentemente-, es en las últimas décadas cuando se intensifica especialmente la deforestación del bosque chaqueño relictual de la zona, debido al aumento de la superficie sembrada y a la diversificación de los cultivos practicados (soja, girasol, maíz, etc.).
}

científico, ordenadas alfabéticamente según este criterio y en renglones subsiguientes se indicaba el o los fitónimos moqoit registrados para dicha especie. El tercer paquete constaba de 227 fichas ordenadas alfabéticamente por nombre científico, las cuales incluían las "especies útiles" -así denominadas por M.C.-. En cada una de ellas se indicaba una somera descripción de uno o más datos etnobotánicos recabados de primera mano entre pobladores moqoit. Además de estos paquetes, la caja citada conservaba una carpeta con hojas mecanografiadas cuyo contenido se dividía en dos partes: una titulada "Arquitectura de la fitonimia mocoví" y otra "Índice de las especies útiles entre los mocovíes del Chaco". Por otro lado, en varias de las fichas correspondientes a especies de la familia de las Fabáceas hay señales que habría consultado a especialistas para su determinación, como en la que consigna: "A.B." y en otras consigna directamente "según Burkart", lo cual indica que aquellas iniciales corresponderían con seguridad al célebre botánico argentino Arturo Burkart, máximo referente argentino en dicha época para tal familia botánica.

La metodología empleada es la correspondiente a la "etnobotánica histórica" que considera a los datos del pasado como una fuente de información primaria, sobre los cuales se aplica el método clásico etnobotánico (ver Rosso, 2012 y Rosso \& Scarpa, 2012). Debido a los avances registrados en la disciplina botánica y en etnobotánica, así como a los cambios culturales y ambientales ocurridos desde que fueron tomados los datos, consideramos que todo abordaje de este tipo debe trascender la simple editorialización y ordenamiento de la información histórica -inédita o publicada-. Esta tendría que ser presentada procurando aplicar -al menosla siguiente metodología: 1) Contextualización espacio-temporal y socio-cultural de los datos; 2) identificaciones botánicas de los taxa referidos; y 3) fitonimia referida y análisis nomenclatural. Por todo ello, los resultados de este trabajo serán estructurados según este criterio.

En todo momento se intentó rescatar la mayor cantidad de datos posibles, por lo cual no solo se incorporan las etnoespecies científicamente determinadas hasta el rango específico, sino también hasta el nivel de género, familia e incluso aquellos fitónimos indígenas sobre los que no se posee identificación alguna. En cuanto a las 
identificaciones botánicas referidas por M.C. se procedió a verificar la validez del binomio, su inclusión como "aceptado" para la flora argentina, su distribución en la provincia del Chaco y su actualización en caso de su condición de "sinónimo", según las bases de datos "Flora del Cono Sur" (Zuloaga \& Morrone, 2009) y "Tropicos" del Missouri Botanical Garden.

Las categorías semánticas estructurales empleadas aquí para clasificar los fitónimos moqoit son las empleadas por Friedberg (1991) y Ellen (2000) -entre otros-: 1) "Lexemas primarios" (nombres propios), como aquéllos que contienen una base nominal o "término básico" -"basic term” según Friedberg (1991)- cuya relación con la planta es puramente arbitraria; 2) "lexemas secundarios", formados por un lexema primario seguido de un calificador de índole "descriptivo" (según remita a propiedades objetivas como color, hábitat, tamaño, etc.), o "alusivo" (si refieren a propiedades no objetivas, tales como términos de parentesco, número, pluralidad, similitud, etc.) y 3) "nombres metafórico-descriptivos", consistentes en frases nominales que indican asociaciones con diferentes tipos de atributos de la planta, tales como morfológicos, utilitarios, perceptuales, fenológicos, de hábitat y de relación con la fauna, entre otros. En la traducción de los lexemas secundarios y descriptivos nos guiamos en parte por lo expresamente registrado por MC. en sus fichas y por el diccionario de Buckwalter \& Litwiller de Buckwalter (2001).

\section{Resultados}

\section{Contextualización espacio-temporal y etnohistórica de los datos}

Según la contrastación realizada entre los datos referidos en los manuscritos hallados y los publicados sobre los moqoit por Martínez Crovetto (1967; 1968a; 1968b; 1968c y 1970), podemos colegir que aquellos habrían sido registrados a campo -al menos- desde el año 1966.

Esto deducimos en función de la fecha de su primer trabajo publicado sobre esta etnia y al hecho de que, en enero de 1967, ya declaraba haber registrado un total de 327 de sus fitónimos. Asimismo, las informaciones habrían sido registradas hasta el año 1967, debido a la última fecha de los datos declarados en sus fichas y a que los demás trabajos fueron publicados durante del primer semestre de 1968.

Respecto a los asentamientos moqoit cuyos representantes brindaron estos datos, todas sus publicaciones al respecto coinciden en afirmar que se trata de aquellos ubicados en el centro-sur de la provincia del Chaco cercanos a las localidades de Charata (Colonia Gral. Necochea), Villa Angela y Villa Berthet. A este respecto, es especialmente ilustrativa su publicación titulada "Estado actual de las tribus mocovíes del Chaco" donde detalla la localización de este pueblo, así como su etnohistoria reciente en los siguientes términos: "A principios de este siglo $[\mathrm{XX}]$, un grupo de indios mocovíes, compuesto por unos 500 individuos de ambos sexos, dejó la región santafecina de San Javier, lugar de su origen y se internaron entre los montes casi inexplorados del centro de la actual provincia del Chaco. Durante más de 20 años vivieron como nómades, hasta que, hacia el año 1922, decidieron establecerse definitivamente en las vecindades de la joven localidad de Charata, mientras hacían venir de Santa Fe a sus parientes y amigos, aprovechando el auge económico promovido por el cultivo del algodón" (Martínez Crovetto, 1968b: 1). A pesar de que este desplazamiento poblacional sería de larga data, los autores tuvimos la oportunidad de observar en una de las localidades referidas (Colonia Gral. Necochea) durante el año 2010 la presencia de algunos descendientes originarios de las riberas del río Paraná (donde se ubica la localidad citada de San Javier).

\section{Identificaciones botánicas}

2.1. Colecciones botánicas: Las únicas referencias que pudimos hallar respecto a la existencia de material vegetal colectado por el autor son ciertas anotaciones del tipo "M678" que invariablemente figuran en las fichas correspondientes a taxones que M.C. no pudo identificar o en aquellas determinaciones a nivel de género. Según el Ing. Krapovickas (com. pers.) este código indica el número de muestra herborizada por dicho autor, quien la habría depositado en el Herbario del IBONE (CTES). Esta observación resulta congruente con lo señalado por Arenas (1992) respecto al hábito de M.C. de coleccionar a campo solo aquellas plantas que le resultaban desconocidas. 


\section{G. F. Scarpa y C. N. Rosso - La etnobotánica moqoit inédita de Raúl Martínez Crovetto}

\subsection{Identificaciones de Martínez Crovetto:}

En una ficha manuscrita en la que contabiliza toda la información indica que el número de especies identificadas fue de 381, de las cuales 61 fueron determinadas hasta género y 33 especies quedaron sin clasificar. De esto se deduce que se trata de un conteo desactualizado ya que en sus trabajos publicados (Martínez Crovetto, 1968c; 1970) registra como "número de especies que los mocovíes conocen" un total de "475". El hecho de que este último conteo coincida con el número de fichas encontradas por nosotros en el segundo paquete rotulado "Latino-mocoví", reafirma que la autoría de todas ellas corresponde a M.C.

Sin embargo, según los datos derivados de nuestro análisis de las fichas, M.C. distingue en total 457 entidades botánicas, de las cuales 5 son hongos, 4 líquenes y 448 son plantas vasculares (segunda fila, Tabla 1). De estas, 378 (84 \%) son identificadas a nivel de especie (4 de ellas a nivel varietal); 49 (11\%) a nivel de género; $13(3 \%)$ a nivel de familia botánica y otras $8(2 \%)$ han quedado sin identificar (en concordancia con el criterio de colección de M.C. antes señalado, consideramos a priori que no habría superposición entre los ejemplares de un mismo género ni de una misma familia herborizados por el autor).

\subsection{Análisis de las identificaciones históricas:} Entre las determinaciones botánicas de M.C. (a excepción de las correspondientes a los hongos y líquenes) se comprobó la existencia de binomios inválidos o inexistentes (como Withfordia elegans), de otros considerados como "no aceptados" para la flora argentina o cuya distribución no se incluye dentro de la provincia fitogeográfica Chaqueña, así como denominaciones que en la actualidad son consideradas como "sinónimos". Entre los primeros figuran "Borreria baselloides" (que consideramos se trataría de Anredera cordifolia (Ten.) Steenis -Basellaceae- ya que su antigua denominación -Boussingaultiagracilis Miers var.pseudobaselloides (Hauman) L.H. Bailey- recuerda a ella y porque su nombre moqoit -nowelá [trepadora]- se corresponde con su forma de vida); "Acalypha guaranitica" (la cual derivaría de manera errónea de $A$. communis Müll. Arg. var. guaranitica Chodat \& Hassl., hoy $A$. communis Müll. Arg., muy común en la provincia del Chaco); "Digitaria penniciligera"; "Polypodium polypodioides" y "Solanum aff. insidiosum", cuyas identidades botánicas se limitaron únicamente a los géneros respectivos. Similar decisión se tomó para "Dioscorea brachybothria" ya que las dos variedades citadas para el país son endémicas de la provincia de Neuquén (las demás son chilenas).

Para todos los nombres científicos aceptados -según la base de datos de la Flora del Cono Surcon distribución chaqueña se procuró precisar, cuando nos fue posible, las identificaciones de M.C. añadiendo taxa subgenéricos. De esta manera se pudo identificar el epíteto específico de algunas plantas determinadas hasta el nivel de género en caso de tratarse de endemismos o por descarte (especies de dicho género presentes en la provincia de Chaco pero no identificadas por M.C.). De la misma manera se procedió en los niveles infraespecíficos (variedades y formas).

Finalmente se proponen como identificaciones "a confrontar" (cf.) los siguientes casos. En primer lugar, MC. indica que los fitónimos indígenas “chiguisé latak'arík" y "chik lateé" corresponden al "palo piedra" y al "palo azul", respectivamente, tal como señala entre paréntesis luego de cada uno de ellos. Estos nombres vulgares hacen referencia, según la fitonimia criolla del Chaco, a Diplokeleba floribunda N.E. Br. (Sapindaceae) y a Cyclolepis genistoides D. Don (Asteraceae) respectivamente, de manera unívoca, por lo cual consideraríamos que se trata de estas especies. Asimismo, el taxón indicado como "Orchidaceae" por M.C. podría corresponder a Sarcoglottis grandiflora (Hook.) Klotzsch (orquídea terrestre chaqueña de vistosas flores color salmón en inflorescencia terminal), debido a que su nombre vernáculo "pelák letáa" ("padre de la achira"), asociaría su hábito de crecimiento, disposición de sus hojas y de sus flores y el color de estas últimas con las de Canna indica $\mathrm{L}$. ("achira"), también común en el Chaco. Por último, el taxón denominado "satachík" sin identificar por M.C., podría corresponder a Maytenus vitis-idaea Griseb., debido a que presenta idéntico nombre en idioma qom (toba) - misma familia lingüística (guaycurú)- según Martínez (2009) y a que su "k" final denota que se trata de una planta leñosa, según demostrara Rosso (2010), al igual que el taxón referido. En suma, de las 448 identificaciones botánicas analizadas (plantas vasculares), se actualizaron $\mathrm{y} / \mathrm{o}$ precisaron la tercera parte de los mismos $(149,33 \%)$.

En el Apéndice (Tabla 1) se enumeran los 
nombres científicos actualizados y los referidos textualmente por M.C. ordenados por familia botánica, a fin de distinguir claramente los datos provenientes de la información histórica primaria de aquellos derivados de la tarea de los autores. Como resultado del análisis realizado las identificaciones botánicas en el Apéndice llegan a un total de 443 taxa, de los cuales 5 son hongos, uno es liquen y 437 son plantas vasculares. Un total de 390 de estas últimas fue identificado a nivel de especie, 47 a nivel de género y 6 a nivel de familia botánica. El $95 \%$ de estas plantas (401) son nativas o asilvestradas al área chaqueña, mientras que sólo 22 resultaron exóticas (la mayoría cultivos moqoit).

\section{3) Fitonimia moqoit}

En la Tabla 1 se detallan 295 fitónimos moqoit a los que aluden las especies vegetales referidas por M.C. Aunque en ocasiones se ha registrado más de un nombre vulgar para una misma entidad botánica, la situación inversa suele ser lo más frecuente, tal como lo sugiere la superioridad numérica de estas últimas respecto de los primeros. Así, las correspondencias totales entre uno y otro tipo de nomenclatura suman 479 datos etnobotánicos. En varios casos se consigna la traducción castellana de los fitónimos indígenas, las cuales son producto tanto de nuestra autoría como de M.C.

\subsection{Notación fonética: En la transcripción} fonética de los fitónimos moqoit M.C. emplea como notación los siguientes fonemas diacríticos: "ㅁ”, el cual correspondería a la "b" (fricativa

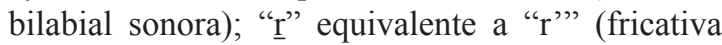
postvelar o uvular sonora) -según Viegas Barros (1995)-; “k” equivalente a "k"'(oclusiva velar sorda glotalizada), "y" a una semiconsonante palatal y "ê" correspondiente a un valor vocálico intermedio entre "e" y "i" (que aquí reproduciremos como "ë"). En el Apéndice (Tabla 1) se reemplaza la notación original por la señalada anteriormente.

\subsection{Clasificación fitonímica de Martínez} Crovetto: En la sección del manuscrito dedicada a la "Arquitectura de la fitonimia mocoví", M.C. refiere prácticamente las mismas categorías de nombres vernáculos por él ya publicadas (Martínez Crovetto, 1968c; 1970). En estas últimas obras se limita a cuantificar los fitónimos incluidos en cada una de ellas (referido entre paréntesis) y a ejemplificarlas con un único nombre indígena. Sin embargo, en el manuscrito hallado se detallan todos los nombres vernáculos incluidos en cada una de las categorías por él definidas, así como los taxa botánicos a los que refieren. Se enumeran a continuación tales categorías junto a la cuantificación indicada en el manuscrito inédito y entre paréntesis en su obra publicada (Martínez Crovetto, 1968c): "Nombres propios: 66" (36); "Nombres derivados de alguna característica de la planta: 7" (17); "Nombres derivados de la utilidad: 22" (2); "Nombres derivados de animales (teniendo en cuenta la semejanza de partes de la planta con órganos de aquellos): 20" (10); "Nombres derivados del uso, real o supuesto que hacen los animales: 26" (14); "Nombres derivados de la semejanza con otras plantas: 14" (13); "Nombres de tipo ecológico: 2" (1); "Nombres híbridos formados por dos palabras, una de las cuales pertenece a otro idioma: 3" (1); "Nombres adaptados de otro idioma: 8" (3); "Nombres de plantas cultivadas: 7" (25) y "Nombres sin ubicación: 18" (4); “Total de nombres: 193" (100). Como se puede apreciar, la cuantificación de las categorías en su carpeta manuscrita supera la que el autor publicara en su momento para cada una de ellas, pero a su vez, resulta inferior al total de fitónimos registrados por él mismo en sus fichas (495 en total). Esto nos permite colegir la existencia de, al menos, tres niveles de actualización de sus datos, cada uno de ellos -quizás- correspondientes a los resultados de sendas campañas, como suele ser usual en las investigaciones etnobotánicas.

La clasificación de la fitonimia en tales categorías resulta en la actualidad extremadamente tipológica y no responde a los criterios formales-estructurales utilizados en el análisis nomenclatural de los estudios etnobiológicos (ya enunciados en la sección Materiales y Métodos). Este análisis meramente referencial realizado sobre la base de clases de nombres descriptivos, subestima la importancia de los lexemas primarios y secundarios ${ }^{4}$ en su sistema fitonímico, al tiempo que sobreestima la de los

\footnotetext{
${ }^{4}$ A excepción de algunos pocos incluídos en la categoría "Nombres derivados de la semejanza con otras plantas", que corresponderían a lo que hoy se consideran "lexemas secundarios".
} 


\section{G. F. Scarpa y C. N. Rosso - La etnobotánica moqoit inédita de Raúl Martínez Crovetto}

nombres descriptivos, es decir, a la inversa de lo que sostienen los enfoques actuales ${ }^{5}$ (Friedberg, 1991; Ellen, 2000). En nuestro país, esta metodología fue utilizada por Scarpa $(2007 ; 2010)$ para los chorote, Martínez (2009) para los qom y Rosso (2010) para los moqoit. A pesar de esto último, su tratamiento de la "arquitectura de la fitonimia mocoví" destaca a M.C. como el primer investigador interesado en nuestro país por este tipo de análisis como parte de estudios etnobotánicos.

3.3. Análisis nomenclatural: Pudieron ser categorizados 276 nombres, ya que del resto (19) se desconoce su pertenencia relativa. En primer lugar se distinguieron los fitónimos indígenas propiamente dichos (262) de los préstamos tomados del castellano (14), ya sean estos re-fonologizados o no. En segunda instancia, a partir de nuestro conocimiento actual de la lengua moqoit y de las traducciones al castellano efectuadas por MC. acerca de muchos de ellos, realizamos un análisis semántico preliminar de la nomenclatura vernácula a los fines de distinguir su pertenencia a cada una de las categorías descriptas en la sección metodológica: lexema primario (NP), secundario (NP2) o metafórico-descriptivo (DE). Los resultados de la cuantificación se grafican en la Figura 1.

\subsubsection{Lexemas primarios: Se identificaron} 84 lexemas primarios, los cuales representan la tercera parte $(32 \%)$ de los nombres moqoit propiamente dichos. De la misma manera que para otros grupos humanos chaquenses (Scarpa, 2010; 2012), las plantas así denominadas constituyen las de mayor significación en su cultura. Se advierte la correspondencia entre la marca léxica " $\mathrm{k}$ " en posición final y la forma de vida leñosa (árboles, arbustos y algunas lianas), fenómeno ya identificado tanto para otras etnias chaquenses

\footnotetext{
5 No se incluyen en estos abordajes de la fitonima aquellos de índole lingüístico-gramatical que analizan la estructura morfemática de los nombres vulgares de las plantas, así como los mecanismos de formación de palabras y otros aspectos, ya que escapan a los fines estrictamente semánticos del análisis nomenclatural típico de la etnobiología. En efecto, el concepto de "lexema" aquí considerado engloba a varias de las categorías derivadas de este último tipo de análisis (nombres simples, compuestos o frases).
}

(Scarpa 2010; 2012) como para los mismos moqoit (Rosso 2010). También se advierte en estos nombres la presencia de genéricos asociados a la forma de vida herbácea -tales como "pewé" (hierba, yuyo)-, graminiforme -“(a)wakapi” (pasto)- y lianescente -"nowelá" (enredaderas, lianas)-. Como se aprecia en el Apéndice (Tabla 1), cada una de ellas se emplea para denominar a numerosas especies vegetales, que a su vez poseen también un nombre específico.

3.3.2. Lexemas secundarios: Se identificaron 37 nombres de este tipo, los que representan el 14 $\%$ de los nombres moqoit. Los calificadores que acompañan a los lexemas primarios con mayor frecuencia de aparición son: "lateé" ("madre") -i.e. "(no)k'olá lateé" "madre de Guadua paraguayana Döll”, para nombrar a Guadua chacoensis (Rojas) Londoño \& P.M. Peterson- y "letaá" ("padre") -i.e. "netarguék letaá", "padre de Prosopis kuntzei Harms", para nombrar a Senna rigida (Hieron.) H.S. Irwin \& Barneby-. La relación entre las plantas a las que estos nombres aluden y aquellas referidas por los lexemas primarios que los conforman, no resulta del todo clara. Sin embargo, en consonancia con lo observado en la fitonimia qom -misma familia lingüística-, se podría hipotetizar que la relación de parentesco se aplica a plantas afines morfológicamente aunque de menor valor y/o tamaño relativo que la nombrada con el lexema primario (como se aprecia en el último ejemplo). Otros lexemas secundarios responden a atributos organolépticos asociados a lexemas primarios de tipo genérico, tales como -i.e. "pewé lalaGaraík" ("yuyo blanco"), para nombrar a Pterocaulon alopecuroides (Lam.) DC. ${ }^{6}$ debido al color blanquecino del envés de sus hojas. Se destaca la ausencia de calificadores que denoten una relación anómala, distorsionada o "falsa" entre la planta nombrada con dicho lexema secundario y la referida por el primario al que hace referencia, tal como ocurre en la fitonimia de los indígenas qom (calificador: "póleo") (Scarpa \& Arenas, 2004) y en la de los chorotes (calificador: “tiój / tók") (Scarpa, 2010).

\footnotetext{
${ }^{6}$ Sólo en dos casos se observó un calificativo que denota "apéndice" de otra planta, tales como maapik naßol (barba del algarrobo), para referirse a Usnea barbata.
} 
3.3.3. Nombres metafórico-descriptivos: Se identificó un total de 141 de estos fitónimos, los cuales representan el $54 \%$ de los nombres indígenas. Los tipos de descripciones más utilizadas aluden a partonomías del tipo "parte de un animal X" (i.e. "layor-r'ái lkaít", lit. "cola del oso hormiguero" para nombrar a los helechos en virtud de sus frondes aladas) o de un personaje mítico (i.e. "k’onasé laañík", lit. "soga de la mujer duende del monte" para Microgramma vacciniifolia (Langsd. \& Fisch.) Copel.). Otras refieren a relaciones planta-animal del tipo "su comida" $-\mathrm{o}$ "donde come"- (i.e. "k'ochiñí lokó"”, lit. "comida de la charata" para Erythroxylum argentinum O.E. Schulz); "su guarida" (i.e. "(na)k’aték lashík", lit. "guarida de la abeja lechiguana", para especies del género Schinus), "su remedio" (i.e. "piók' latarík", lit. "remedio del perro" para Cestrum parqui L'Hér.); "su amuleto" (i.e. "nasaló nemaík", lit. "amuleto de la perdiz" para especies del género Polygala). También suelen nombrar a algunas plantas en función de atributos organolépticos de tipo gustativo (i.e. "chemmak'-raík", lit. "picante" para especies del género Capsicum); colorimétrico (i.e. "togué' le'noßiar'ái", lit. "flor colorada" para Eupatorium macrocephalum Less.), háptico (i.e. "pok'oík", lit. "que quema" para las plantas urticantes) o morfológico (i.e. "lo'ió", lit. "gorda", "carnosa" para Portulaca oleracea L.). Tanto por la cantidad de las descripciones que incluyen como por el valor que estas representan, estos tipos de nombres aportan sustanciales datos para

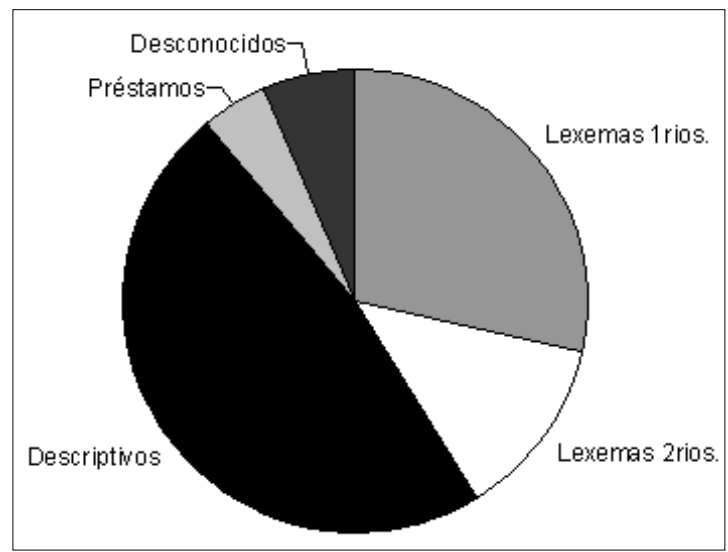

Fig. 1. Categorías semánticas estructurales de la fitonimia moqoit. profundizar acerca de las significaciones de las plantas en base a tales indicios.

3.3.4. Préstamos de otras lenguas: Se distinguen los "préstamos de traducción" al idioma indígena de fitónimos criollos de aquellos "préstamos absolutos" que han sido re-fonologizados. Entre los primeros se cuentan "shipr'ák' lk'aiít" -lit. "cola de caballo", Equisteum giganteum L.) o "naiiapëk lasoté" -lit. "cuerno del diablo", Ibicella lutea (Lindl.) Van Eselt.), entre otros. Respecto a los absolutos podemos mencionar "akós" (por "ajo", Allium sativum L.); "manyóka" (por "mandioca", Manihot esculenta Crantz), "chirígo" (por "trigo", Triticum aestivum L.); "álpa" (por "alfa / alfalfa"; Medicago sativa L.), "ladásna" (por "durazno", Prunus persica (L.) Batsch), "narankík” (por “naranjín”, Citrus sinensis (L.) Osbeck).

\subsection{Fitónimos sin correspondencia botánica:} De las nueve (9) entidades botánicas sin identificar mencionadas tres (3) corresponden a líquenes y a un hongo cuyos nombres moqoit se incluyen en el Apéndice (Tabla 1). El resto de los fitónimos con sus respectivas etiquetas son: "salok'llík" ( ${ }^{\circ}$ 75; 76); "ler'ra" ("espinoso") (M677); "nowair'á nemaík" (lit. "amuleto del zorro o aguará") (M680); "piók' latarík lateé" ("madre del remedio del perro") (M710) y "lak'abiar'aík lateé" ("madre del quebradizo"). A excepción del primero de ellos -que desconocemos su categoría-, los otros cuatro (4) corresponden a nombres metafóricodescriptivos.

\section{Discusión y Conclusiones}

Se advierte en primer término el gran volumen de nombres indígenas recabados (295) y de identidades botánicas a los que estos hacen referencia (443), en comparación con los antecedentes ya citados para la fitonimia moqoit (no debe confundirse esta cuantificación con la cantidad de taxa que M.C. afirma haber registrado -475-, ni con los que efectivamente dicho autor registró según nuestro conteo -457-). El número de nombres indígenas registrados por M.C. supera sustancialmente los obtenidos para etnias chaquenses del Chaco semiárido de la provincia de Salta (198 entre los chorote-iyojwa'ja - para 238 taxa botánicos- y 


\section{G. F. Scarpa y C. N. Rosso - La etnobotánica moqoit inédita de Raúl Martínez Crovetto}

214 para los chorote-iyowujwa -para 225 taxa-) (Scarpa, 2010). Sin embargo, resultan comparables a los registrados 40 años después para los qombermejeños del Chaco Central de la provincia del Chaco (250 fitónimos -para 235 taxa-) (Martínez, 2009), aunque para un número mucho mayor de taxa vegetales (443). Estas diferencias se explicarían tanto por razones de índole ecológica-diversidad vegetal comparable-, como por su pertenencia a la misma familia lingüística - guaycurú- de la última etnia citada (a diferencia de los chorote del Chaco semiárido que pertenecen a la familia lingüística mataco-mataguayo).

Por otra parte, se registran 479 correspondencias entre taxa botánicos y nomenclatura indígena, cada una de las cuales constituyen datos etnobotánicos propiamente dichos.

La clasificación de la fitonimia en función de las categorías semánticas indica un neto predominio de nombres descriptivos (54\%), los cuales superan la cantidad de lexemas primarios (33\%) y secundarios (14\%) (Fig. 1). La importancia de estos resultados radica en sus implicancias etnotaxonómicas, en cuanto a que los lexemas primarios (i.e. "algarrobo") suelen cumplir el rol de (etno-)géneros y los secundarios el de etnoespecies (i.e. "algarrobo blanco") en los sistemas de clasificación vernáculos. Así, la relación entre las dos últimas categorías, que indica la proporción de (etno-)géneros monotípicos, resulta sustancialmente más baja -19 \%- que la registrada para otros grupos chaquenses, como los chorotes-iyojwa'ja (31 \% -50 \% lexemas primarios y $19 \%$ secundarios-) y los chorote-iyowujwa (29\% $-52 \%$ y $23 \%$-) (Scarpa, 2010). Paradójicamente, los datos registrados entre criollos del Chaco semiárido por categoría semántica (34\% primarios, $18 \%$ secundarios y $48 \%$ descriptivos) y la proporción de (etno-)géneros monotípicos (16 \%) (Scarpa, 2012), fueron semejantes a los hallados, aunque estos no serían atribuibles a cuestiones de préstamo cultural.

Otras implicancias de los resultados obtenidos estriban en que muchos de los nombres genéricos registrados por M.C. corresponden a verdaderas categorías etnoclasificatorias denominadas "formas de vida", según la terminología etnotaxonómica empleada por Berlin (1992) para cientos de pueblos alrededor del mundo. Estos nombres genéricos responden al criterio clasificatorio definido según atributos fisonómicos de la vegetación, tales como "herbáceas" ("pewé", lit. hierba, yuyo), "graminiformes"(“(a)wakapi", lit. pasto) y "lianescente" ("nowelá", lit. enredaderas, lianas), razón por la cual son así denominadas un gran número de especies botánicas.

Al comparar la exhaustividad de nombres indígenas aquí consignados con la relativa escasez de datos sobre usos y significaciones registrados en estos mismos manuscritos inéditos ${ }^{7}$, se aprecia una evidente desproporción entre ambas. Esta estructura y distribución de los datos etnobotánicos resultan típicas de los que se esperarían encontrar durante las primeras fases de una investigación etnobotánica de largo alcance (la cual habría quedado trunca).

Además de la importancia de los datos aquí referidos para el patrimonio cultural moqoit, estos resultan especialmente valorables por el hecho de haber sido documentados hace ya 45 años. Esto implica que existen bajas probabilidades de volver a registrarlos a campo en la actualidad debido a: i) El cambio ambiental operado como consecuencia de los altos niveles de deforestación existentes en los asentamientos moqoit actualmente sometidos a explotación agrícola intensiva; ii) las profundas transformaciones culturales operadas en el seno de la sociedad indígena desde dicha época hasta la actualidad; iii) los traslados masivos de las poblaciones moqoit desde otras zonas fitogeográficas ocupadas desde antaño (ribera paranaense), lo cual redunda en el registro de ciertas especies vegetales ajenas a su ámbito actual de residencia. Entre éstas pueden mencionarse las que habitan en las "selvas de ribera" o "en galería" del río Paraná y sus afluentes, tales como Ficus luschnathiana (Miq.) Miq., Eugenia uniflora L., Allophyllus edulis (A. St.Hil., A. Juss. \& Cambess.) Hieron. Ex Niederl., así como algunas plantas acuáticas tales como el "irupé" (Victoria cruziana Orb.).

Por último, consideramos que sería un grave error desdeñar los datos etnobotánicos registrados por M.C. por el hecho de no haber coleccionado ejemplares de herbario en compañía de los mocovíes, debido a: i) La existencia de material estudiado por M.C. de cada una de las especies mencionadas -en otra circunstancia y lugar-,

\footnotetext{
7 Estos datos, que serán publicados en la revista Bonplandia 23(2), se incluyen en el tercer paquete de fichas denominado "Especies útiles", correspondientes al mismo trabajo de campo del autor a partir del cual hubo de registrar los fitónimos considerados en este artículo.
} 
legado al Herbario del Instituto de Botánica del Nordeste (IBONE) bajo la sigla CTES, donde se encuentran depositados; ii) la confiabilidad que ameritan sus determinaciones botánicas por su amplio conocimiento sobre la flora del Chaco Oriental, el cual ha sido demostrado tanto en sus trabajos botánicos y etnobotánicos sobre esta región, como por su calidad de profesor titular en Botánica Sistemática y Fitogeografía en la Universidad Nacional del Nordeste; iii) al aval explícito que M.C. habría conferido a estas determinaciones botánicas inéditas, como lo demuestra la publicación de las mismas cuantificaciones de especies y fitónimos referidos en este trabajo (Martínez Crovetto, 1968c) y iv) la comprobación en 70 (setenta) oportunidades de idénticas correspondencias taxa botánicos / etnoespecies entre las registradas por M.C. y las obtenidas a campo por los autores -hasta el momento- en nuestros resultados preliminares sobre la etnobotánica de esta etnia (Rosso \& Scarpa, inédito). Estos últimos fueron documentados sobre la base de ejemplares botánicos depositados en el Herbario del MACN (BA) que sí fueron coleccionados en compañía de representantes moqoit en los mismos asentamientos visitados por aquél.

La cuantiosa información histórica en términos de nombres indígenas recopilados y de taxa botánicos a los que hacen referencia que se expone como resultados de este trabajo contextualizada en tiempo y espacio, pone de manifiesto en forma indudable la relevancia que las inéditas investigaciones de M.C. tienen todavía para la etnobotánica argentina actual.

\section{Agradecimientos}

Al Ing. Antonio Krapovickas por facilitarnos los materiales de trabajo de Raúl Martínez Crovetto archivados en la Biblioteca del Instituto de Botánica del Noreste, Facultad de Ciencias Agrarias de la UNNE, Corrientes. Al Consejo Nacional de Investigaciones Científicas y Tecnológicas.

\section{Bibliografía}

ARENAS, P. 1992. Homenaje. Raúl Nereo Martínez Covetto (1921-1988), su contribución a la etnobotánica y a los estudios americanistas. Parodiana 5(2): 505-519.
BERLIN, B. 1992. Ethnobiological classification. Principles of categorization of plants and animals in traditional societies. Princeton University Press, Princeton.

BUCKWALTER, A. \& L. LITWILLER de BUCKWALTER. 2001. Vocabulario Mocoví. Ediciones de autor, Formosa.

ELLEN, R. 2000. The cultural relations of classification. An analysis of Nuaulu animal categories from Central Seram. Cambridge University Press, Cambridge.

FRIEDBERG, C. 1991. Operative aspects of folk classification. In: PAWLEY, A. (ed.), Man and a Half. Essays in Pacific Anthropology and Ethnobiology in Honour of Ralph Bulmer, pp. 102-109. The Polynesian Society, Auckland.

GUALDIERI, B. 2006. Clasificadores guaycurúes: Un desafío para la lingüística. En: FERNÁNDEZ GARAY, A. \& M. MALVESTITTI (eds.), Estudios lingüisticos y sociolingüisticos de lenguas indígenas sudamericanas, pp. 1-18. Universidad de La Pampa, Santa Rosa.

MARTÍNEZ, G. 2009. Fitonimia de los Tobas Bermejeños (Chaco Central, Argentina). Hacia una nueva carta étnica del Gran Chaco 8: 194-212.

MARTÍNEZ CROVETTO, R. 1967. Contribución al estudio de la cerámica actual de los indios mocovíes del Chaco (República Argentina). Etnobiológica 1: 1-7.

MARTÍNEZ CROVETTO, R. 1968a. Viejos juegos de los indios mocovíes. Etnobiológica 2: 1-31.

MARTÍNEZ CROVETTO, R. 1968b. Estado actual de las tribus mocovíes del Chaco (República Argentina). Etnobiológica 7: 1-33.

MARTÍNEZ CROVETTO, R. 1968c. Introducción a la etnobotánica del Nordeste Argentino. Etnobiológica 11: 1-10.

MARTÍNEZ CROVETTO, R. 1970. La etnobotánica de los grupos aborígenes del nordeste argentino. Bol. Soc. Arg. Bot. 11(Supl.): 211-215.

PAUCKE, F. 1942-1944. Hacia allá y para acá. Una estadía entre los indios Mocobies, 1749-1767. Volumen I, II y III. Universidad de Tucumán, Tucumán.

PIRONDO, A. \& H. A. KELLER. 2012. Raúl N. Martínez Crovetto: los albores de la etnobotánica en la Argentina. Introducción a Estudios Etnobotánicos V. Bonplandia 21(2): 101-107.

ROSSO, C. N. 2010. Compilación y análisis preliminar de la fitonimia de la flora leñosa demcomunidades mocovíes del sudoeste chaqueño. En: MESSINEO, C., G.F. SCARPA \& F. TOLA (comp.), Léxico y categorización etnobiológica en grupos indígenas del Gran Chaco, pp. 251-271. Universidad Nacional de La Pampa, Santa Rosa. 


\section{G. F. Scarpa y C. N. Rosso - La etnobotánica moqoit inédita de Raúl Martínez Crovetto}

ROSSO, C. N. 2012. La etnobotánica de los grupos mocovies de la reducción de San Javier, en el Gran Chaco, durante el siglo XVIII. Tesis doctoral Facultad de Filosofía y Letras, Universidad de Buenos Aires, Buenos Aires.

ROSSO, C.N. \& G. F. SCARPA. 2012. Identificaciones botánicas de las plantas empleadas entre los mocovíes en la reducción de San Javier durante el siglo XVIII a partir de la obra de Florián Paucke, S. J. En: ARENAS, P. (ed.), Etnobotánica en zonas áridas y semiáridas del Cono Sur de Sudamérica, pp. 45-70. Edición CEFYBO-CONICET, Buenos Aires.

SCARPA, G. F. 2007. Hacia una etnotaxonomía vegetal Chorote I: Fitonimia, sistema nomenclatural y comparación dialectal. Suplemento Antropológico 42(1): 81-119.

SCARPA, G. F. 2010. Hacia una etnotaxonomía vegetal chorote II: Clasificación de las plantas entre las parcialidades iyojwá'ja y iyowújwa del Chaco argentino. En: MESSINEO, C., G. F. SCARPA \& F. TOLA (comps.), Léxico y categorización etnobiológica en grupos indigenas del Gran Chaco, pp. 157-198. Universidad Nacional de La Pampa, Santa Rosa.

SCARPA, G. F. 2012. "Palos, yuyos, pencas, bejucos y pastos": Los nombres de las plantas y su clasificación etnobotánica por los criollos del Chaco Semiárido Norte (NE Salta - W Formosa). En: ARENAS P. (ed.), Etnobotánica en zonas áridas y semiáridas del Cono Sur de Sudamérica, pp. 117-144. Ed. Sigma, Buenos Aires.
SCARPA, G. F. \& P. ARENAS. 2004. Vegetation units of the Argentine Semiarid Chaco: The Toba-Pilagá perception. Phytocoenologia 34(1): 133-161.

SCARPA, G. F. \& C. N. ROSSO. 2011. Etnobotánica del "coro" (Nicotiana paa, Solanaceae): Un tabaco silvestre poco conocido del extremo sur de Sudamérica. Bonplandia 20 (2): 391-404.

TROPICOS.ORG. 2009. Missouri Botanical Garden. Disponible en: http://www.tropicos.org. [Acceso: 28 April 2014]

VIEGAS BARROS, J. P. 1995. Zoonimia y etnozoología de los Pilagá, Toba, Mocoví, Mataco y Vilela. Raúl N. Martínez Crovetto. Universidad de Buenos Aires, Instituto de Lingüística, Facultad de Filosofía y Letras, Buenos Aires.

ZAPATA GOLLÁN, A. 1945. Nomenclatura mocobí de animales y plantas. Boletín delDepartamento de Estudios Etnográficos y Coloniales 1: 51-62.

ZULOAGA, F. \& O. MORRONE. 2009. Flora del Cono Sur. Catálogo de las Plantas Vasculares. Instituto de Botánica "Darwinion", Buenos Aires. Disponible en: http://www2.darwin.edu.ar/Proyectos/ FloraArgentina/FA.asp. [Acceso: 28 April 2014]

Recibido el 26 de mayo de 2014, aceptado el 23 de septiembre de 2014 . 
Bol. Soc. Argent. Bot. 49 (4) 2014

Tabla 1: Apéndice. Identificaciones botánicas actualizadas y de M.C., fitonimia moqoit y categorias nomenclaturales. (*): Especies exóticas. NP: Lexemas primarios (nombres propios); NP2: Lexemas secundarios; DE: Nombres metafórico-descriptivos; PRE: Préstamos.

\begin{tabular}{|c|c|c|c|}
\hline Especie actual & Especie M.C. (lit.) & Fitónimo moqoit (castellano) & Tipo \\
\hline \multicolumn{4}{|l|}{ FUNGI } \\
\hline AGARICACEAE & Agaricáceos & shiprák' notosé (glande del caballo) & $\mathrm{DE}$ \\
\hline AURICULARIACEAE & Gasteromycetes & wakañí lalék (bosta de las estrellas) & $\mathrm{DE}$ \\
\hline Auricularia polytrichia (Mont.) Sacc. ${ }^{*}$ & Auricularia polytrichia & k'opák Ikelá (oreja de palo) & $\mathrm{DE}$ \\
\hline \multicolumn{4}{|l|}{ POLYPORACEAE } \\
\hline Panus sp. & Panus sp. & k'onasé Ikelá (oreja de la diabla del monte) & $\mathrm{DE}$ \\
\hline $\begin{array}{l}\text { Pycnoporus sanguineus } \\
\text { (L. ex Fr.) Murray }\end{array}$ & Pycnoporus sanguineus & k'opák Ikelá (oreja de palo), Ikelá & $\mathrm{DE}$ \\
\hline$?$ & Withfordia elegans & k'opák Ikelá (oreja de palo) & $\mathrm{DE}$ \\
\hline \multicolumn{4}{|l|}{ LICHENES } \\
\hline $\begin{array}{l}\text { Usnea barbata (L.) } \\
\text { Weber ex F.H. Wigg }\end{array}$ & Usnea barbata & maapik naßol (barba del algarrobo) & NP2 \\
\hline$?$ & Lichenes corticícolas & ronará nokopák (pegados al palo) & $\mathrm{DE}$ \\
\hline$?$ & Lichenes chatos & loßi (pelecho, cáscara seca) & $\mathrm{DE}$ \\
\hline ? & Lichenes & lesó (cáscara seca), ßal (barba?) & DE \\
\hline \multicolumn{4}{|l|}{ FANEROGAMAE } \\
\hline \multicolumn{4}{|l|}{ ACANTHACEAE } \\
\hline Dyschoriste humilis Lindau & Dyschoriste humilis & ik'olák le’noßiar'ái (flor azul) & $\mathrm{DE}$ \\
\hline Justicia gilliesii (Nees) Benth. & Justicia echegarayi & ik'olák le’noßiar'ái (flor azul) & $\mathrm{DE}$ \\
\hline \multicolumn{4}{|l|}{ ACHATOCARPACEAE } \\
\hline Achatocarpus praecox Griseb. & Achatocarpus praecox & lamk'áik & NP \\
\hline \multicolumn{4}{|l|}{ AGAVACEAE } \\
\hline Yucca gloriosa L. * & Yucca gloriosa & k'atá & ? \\
\hline \multicolumn{4}{|l|}{ ALISMATACEAE } \\
\hline $\begin{array}{l}\text { Echinodorus grandiflorus } \\
\text { (Cham. \& Schltdl.) Micheli }\end{array}$ & Echinodorus grandiflorus & naanók lapotó (poncho del yacaré) & DE \\
\hline $\begin{array}{l}\text { Sagittaria montevidensis Cham. } \\
\text { \& Schltdl. ssp. montevidensis }\end{array}$ & Sagittaria montevidensis & $\begin{array}{l}\text { naanók lapotó (poncho del yacaré); } \\
\text { naanók lkelá (oreja del yacaré) }\end{array}$ & $\begin{array}{l}\mathrm{DE} \\
\mathrm{DE}\end{array}$ \\
\hline \multicolumn{4}{|l|}{ AMARANTHACEAE } \\
\hline Alternanthera pungens Kunth. & Alternanthera pungens & piñái naté' (culo de langosta) & $\mathrm{DE}$ \\
\hline Alternanthera sp. & Alternanthera sp. & repalichák & $?$ \\
\hline Amaranthus hybridus L. hybridus & Amaranthus quitensis & rebaullí & NP \\
\hline Amaranthus muricatus (Moq.) Hieron. & Amaranthus muricatus & $\begin{array}{l}\text { ka'alañé/í (aplastado); piók' lolá letaá } \\
\text { (testículos de perro su padre) }\end{array}$ & $\begin{array}{l}\mathrm{DE} \\
\mathrm{DE}\end{array}$ \\
\hline Amaranthus viridis $L$. & Amaranthus gracilis & rebaullí & NP \\
\hline Gomphrena celosioides Mart. & Gomphrena celosioides & piñái naté' (culo de langosta) & $\mathrm{DE}$ \\
\hline Gomphrena pulchella Mart. & Gomphrena pulchella & nachibirí latá (gusanos su remedio) & $\mathrm{DE}$ \\
\hline $\begin{array}{l}\text { Iresine diffusa Humb. \& Bonpl. } \\
\text { Ex Willd. var. diffusa }\end{array}$ & Iresine celosia & $\begin{array}{l}\text { koñilalá nemaík (amuleto de la } \\
\text { avispa rubita / amarilla) }\end{array}$ & DE \\
\hline $\begin{array}{l}\text { Pfaffia tuberosa (Spreng.) } \\
\text { Hicken f. tuberosa }\end{array}$ & Pfaffia sericea & dak'-rák neñi (bigote de chuña) & $\mathrm{DE}$ \\
\hline \multicolumn{4}{|l|}{ AMARYLLIDACEAE } \\
\hline $\begin{array}{l}\text { Zephyranthes candida } \\
\text { (Herb. ex Lindl.) Herb. }\end{array}$ & Zephyranthes candida & k'alachí (cebolla) & NP \\
\hline \multicolumn{4}{|l|}{ ANACARDIACEAE } \\
\hline Astronium balansaeEngl. & Astronium balansae & anták & NP \\
\hline Schinopsis balansae Engl. & Schinopsis balansae & kotapík & NP \\
\hline Schinopsis lorentzii (Griseb.) Engl. & Schinopsis lorentzii & ñiar'aík & NP \\
\hline
\end{tabular}




\section{G. F. Scarpa y C. N. Rosso - La etnobotánica moqoit inédita de Raúl Martínez Crovetto}

\begin{tabular}{|c|c|c|c|}
\hline Especie actual & Especie M.C. (lit.) & Fitónimo moqoit (castellano) & Tipo \\
\hline $\begin{array}{l}\text { Schinus fasciculatus (Griseb.) } \\
\text { I.M. Johnst. var. fasciculatus }\end{array}$ & Schinus fasciculatus & $\begin{array}{l}\text { (na)k'aték lashík (guarida de } \\
\text { la abeja lechiguana) }\end{array}$ & $\mathrm{DE}$ \\
\hline $\begin{array}{l}\text { Schinus longifolius (Lindl.) } \\
\text { Speg. var. Iongifolius }\end{array}$ & Schinus longifolius & $\begin{array}{l}\text { (na)k'aték lashík (guarida de } \\
\text { la abeja lechiguana) }\end{array}$ & $\mathrm{DE}$ \\
\hline Schinus sp. & Schinus sp. (M675) & $\begin{array}{l}\text { (na)k'aték lashík (guarida de } \\
\text { la abeja lechiguana) }\end{array}$ & DE \\
\hline \multicolumn{4}{|l|}{ ANNONACEAE } \\
\hline Rollinia emarginata Schltdl. & Rollinia emarginata & pelé lokoík (donde come el ciervo) & DE \\
\hline \multicolumn{4}{|l|}{ APIACEAE } \\
\hline $\begin{array}{l}\text { Cyclospermum leptophyllum } \\
\text { (Pers.) Sprague }\end{array}$ & Apium leptophyllum & palók & NP \\
\hline Eryngium coronatum Hook. \& Arn. & Eryngium coronatum & rerón olé (catinguita) & DE \\
\hline Eryngium eburneum Decne. & Eryngium eburneum & palor'ó & NP \\
\hline Eryngium elegans Cham. \& Schltdl. & Eryngium elegans & palor'ó & NP \\
\hline Eryngium horridum Malme & Eryngium horridum & palor'ó & NP \\
\hline $\begin{array}{l}\text { Eryngium sanguisorba } \\
\text { Cham. \& Schltdl. }\end{array}$ & Eryngium sanguisorba & palor'ó & NP \\
\hline APOCYNACEAE & Asclepiadaceae (M709) & loßia'k loeté (teta de tapir) & DE \\
\hline $\begin{array}{l}\text { Araujia angustifolia (Hook. } \\
\text { \& Arn.) Decne. }\end{array}$ & Araujia angustifolia & k'oplak(')ái(k) & NP \\
\hline $\begin{array}{l}\text { Araujia odorata (Hook. \& } \\
\text { Arn.) Fontella \& Goyder }\end{array}$ & Morrenia odorata & loßar'áik & NP \\
\hline Asclepias mellodora A. St.-Hil. & Asclepias mellodora & k'agueretá la/oeté (teta del guazuncho) & DE \\
\hline $\begin{array}{l}\text { Aspidosperma quebracho- } \\
\text { blanco Schltdl. }\end{array}$ & $\begin{array}{l}\text { Aspidosperma } \\
\text { quebracho-blanco }\end{array}$ & nodík / norík & NP \\
\hline $\begin{array}{l}\text { Aspidosperma triternatum } \\
\text { Rojas Acosta }\end{array}$ & Aspidosperma chakensis & $\begin{array}{l}\text { koñiguí (raíz amarilla?); norík letaá } \\
\text { (padre del quebracho blanco) }\end{array}$ & $\begin{array}{l}\text { DE } \\
\text { NP2 }\end{array}$ \\
\hline $\begin{array}{l}\text { Gonolobus rostratus (Vahl) } \\
\text { Roem. \& Schult. }\end{array}$ & Exolobus patens & k'oplak'ái(k) & NP \\
\hline Morrenia stormiana (Morong) Malme & Morrenia stormiana & $\begin{array}{l}\text { k'agueretá loeté lateé (madre } \\
\text { de la teta del guazuncho) }\end{array}$ & DE \\
\hline Oxypetalum arnottianum H. Buek & Oxypetalum arnottianum & k'agueretá la/oeté (teta del guazuncho) & DE \\
\hline $\begin{array}{l}\text { Oxypetalum capitatum } \\
\text { Mart. ssp. mirabile (Malme) } \\
\text { Fontella \& Farinaccio }\end{array}$ & $\begin{array}{l}\text { Oxypetalum } \\
\text { proboscideum }\end{array}$ & k'agueretá la/oeté (teta del guazuncho) & $\mathrm{DE}$ \\
\hline $\begin{array}{l}\text { Oxypetalum parviflorum (Decne.) } \\
\text { Decne. var. parviflorum }\end{array}$ & Oxypetalum parviflorum & k'agueretá la/oeté (teta del guazuncho) & DE \\
\hline Oxypetalum solanoides Hook. \& Arn. & Oxypetalum solanoides & k'agueretá la/oeté (teta del guazuncho) & DE \\
\hline Tweedia brunonis Hook. \& Arn. & Tweedia brunonis & k(')ak(')aré Ikaík (cabeza de carancho) & DE \\
\hline \multicolumn{4}{|l|}{ AQUIFOLIACEAE } \\
\hline $\begin{array}{l}\text { Ilex paraguariensis* A. St.- } \\
\text { Hil. var. paraguariensis }\end{array}$ & Ilex paraguariensis & idaßá & PRE \\
\hline \multicolumn{4}{|l|}{ ARACEAE } \\
\hline Pistia stratiotes L. & Pistia stratiotes & k’ol; re-topí & $\begin{array}{l}\text { NP, } \\
?\end{array}$ \\
\hline \multicolumn{4}{|l|}{ ARECACEAE } \\
\hline Copernicia alba Morong & Copernicia alba & chabik & NP \\
\hline $\begin{array}{l}\text { Trithrinax campestris } \\
\text { (Burmeist.) Drude \& Griseb. }\end{array}$ & Trithrinax campestris & k'atá & NP \\
\hline ASTERACEAE & Compuesta (M669) & $\begin{array}{c}\text { koñilalá nemaík letaá (madre del } \\
\text { amuleto de la avispa rubita) }\end{array}$ & $\mathrm{DE}$ \\
\hline Acanthospermum hispidumDC. & $\begin{array}{l}\text { Acanthospermum } \\
\text { hispidum }\end{array}$ & taasót waká Ikaík (cabeza de vaca) & DE \\
\hline
\end{tabular}




\begin{tabular}{|c|c|c|c|}
\hline Especie actual & Especie M.C. (lit.) & Fitónimo moqoit (castellano) & Tipo \\
\hline $\begin{array}{l}\text { Aldama anchusifolia (DC.) } \\
\text { E.E.Schill. \& Panero }\end{array}$ & Viguiera anchusaefolia & koñíle nowiar'ái-olé (florcita amarilla) & $\mathrm{DE}$ \\
\hline Ambrosia elatior $\mathrm{L}$. & Ambrosia elatior & $\begin{array}{l}\text { pewé chëm (yuyo amargo); } \\
\text { Ipaá (? su tabaco) }\end{array}$ & $\begin{array}{c}\text { NP2 } \\
?\end{array}$ \\
\hline Ambrosia tenuifolia Spreng. & Ambrosia tenuifolia & pewé chëm (yuyo amargo) & NP2 \\
\hline Baccharis articulata (Lam.) Pers. & Baccharis articulata & naták lapelá (pata de gallareta) & $\mathrm{DE}$ \\
\hline Baccharis coridifolia DC. & Baccharis coridifolia & piók' nkoronak'jlá' (donde orina el perro) & DE \\
\hline Baccharis dracunculifolia DC. & Baccharis dracunculifolia & noßogué lateé (chilca madre) & NP2 \\
\hline Baccharis notosergila Griseb. & Baccharis notosergila & milgrát; mißiát & NP \\
\hline Baccharis sp. & Baccharis sp. (M719) & noßogué / nowogué & NP \\
\hline Baccharis vulneraria Baker & $\begin{array}{l}\text { Baccharidastrum } \\
\text { triplinervium }\end{array}$ & koiók & NP \\
\hline Bidens pilosa $\mathrm{L}$. & Bidens pilosa & ronái- olé (que se clava o adhiere) & DE \\
\hline $\begin{array}{l}\text { Bidens subalternans DC. } \\
\text { var. subalternans }\end{array}$ & Bidens subalternans & ronái- olé (que se clava o adhiere) & DE \\
\hline $\begin{array}{l}\text { Campuloclinium macrocephalum } \\
\text { (Less.) DC. }\end{array}$ & $\begin{array}{l}\text { Eupatorium } \\
\text { macrocephalum }\end{array}$ & $\begin{array}{l}\text { nawenék lokoík (donde come el venado); } \\
\text { togué' le'noßiar'ái (flor colorada) }\end{array}$ & $\begin{array}{l}\mathrm{DE} \\
\mathrm{DE}\end{array}$ \\
\hline cf. Cyclolepis genistoides D. Don & "palo azul" & chik-lateé (azul el padre) & $\mathrm{DE}$ \\
\hline Chaptalia nutans (L.) Pol. & Chaptalia nutans & shiraigó lkaík (cabeza de luna) & DE \\
\hline Chaptalia sinuata (Less.) Baker & Chaptalia sinuata & lalar'ái lawé (hoja blanca) & DE \\
\hline $\begin{array}{l}\text { Chromolaena christieana } \\
\text { (Baker) R.M. King \& H. Rob. }\end{array}$ & Eupatorium christeanum & ik'olák le’noßiar'ái (flor azul) & DE \\
\hline Conyza bonariensis (L.) Cronquist & Conyza bonariensis & pewé nawerelék (yuyo que ennegrece) & NP2 \\
\hline Eclipta prostrata (L.) L. & Eclipta alba & tak'ák' lechí (pata de chajá) & DE \\
\hline Elephantopus mollis Kunth & Elephantopus mollis & nawenék nemaík (amuleto del venado) & $\mathrm{DE}$ \\
\hline Eupatorium sp. & Eupatorium sp. & ik'olák le'noßiar'ái (flor azul) & $\mathrm{DE}$ \\
\hline Eupatorium sp. & Eupatorium sp. & togué' Ie'noßiar'ái (flor colorada) & DE \\
\hline $\begin{array}{l}\text { Gaillardia megapotamica (Spreng.) } \\
\text { Baker var. radiata (Griseb.) Baker }\end{array}$ & Gaillardia megapotamica & mañik halók (comida del suri) & $\mathrm{DE}$ \\
\hline Gamochaeta filaginea (DC.) Cabrera & Gamochaeta filaginea & $\begin{array}{l}\text { ronái- olé (que se clava o } \\
\text { adhiere); pewé (yuyo) }\end{array}$ & $\begin{array}{l}\text { DE } \\
\text { NP }\end{array}$ \\
\hline Gamochaeta sp. & Gamochaeta sp. (M694) & noomr'á letaá & $?$ \\
\hline Mikania micrantha Kunth & Mikania micrantha & $\begin{array}{l}\text { pa(')lotr'áik (¿?);nak'atek Ikolák } \\
\text { (donde chupa la abeja lechiguana) }\end{array}$ & $\begin{array}{l}\mathrm{NP} \\
\mathrm{DE}\end{array}$ \\
\hline Mikania sp. & Mikania sp. (M665) & palotr'áik letaá & NP2 \\
\hline Mikania sp. & Mikania sp. (M696) & nowelá nawaté & NP2 \\
\hline Mikania sp. & Mikania sp. (M713) & pa(')lotr'áik & NP \\
\hline Parthenium hysterophorus L. & $\begin{array}{l}\text { Parthenium } \\
\text { hysterophorus }\end{array}$ & pewé (yuyo) & NP \\
\hline Picrosia longifolia D. Don & Picrosia longifolia & $\begin{array}{l}\text { shilkáik nemáik (amuleto de } \\
\text { la iguana / lagatija) }\end{array}$ & $\mathrm{DE}$ \\
\hline Pluchea sagittalis (Lam.) Cabrera & Pluchea sagittalis & $\begin{array}{l}\text { wakasñí nedawá (yerba del lucero); ne(te) } \\
\text { tr'át naraasé (que bebe el lucero) }\end{array}$ & $\begin{array}{l}\text { NP2 } \\
\text { DE }\end{array}$ \\
\hline Porophyllum ruderale (Jacq.) Cass. & Porophyllum ruderale & ronái- olé (que se clava o adhiere) & $\mathrm{DE}$ \\
\hline $\begin{array}{l}\text { Pterocaulon alopecuroides } \\
\text { (Lam.) DC. }\end{array}$ & $\begin{array}{l}\text { Pterocaulon } \\
\text { alopecuroides }\end{array}$ & pewé lalar'araík (yuyo blanco) & NP2 \\
\hline Pterocaulon lorentzii Malme & Pterocaulon lorentzii & noomr'á & NP \\
\hline Pterocaulon polystachyum DC. & Pterocaulon polystachium & toró nemáik (amuleto del toro) & $\mathrm{DE}$ \\
\hline Senecio grisebachii Baker & Senecio grisebachii & $\begin{array}{l}\text { la/er'á nalok' r'uá (nacidos el mismo } \\
\text { día que el "chaito" -sic-) }\end{array}$ & DE \\
\hline Senecio sp. & Senecio sp. (M705) & k'ochiñí lokoík (donde come la charata) & DE \\
\hline Solidago chilensis Meyen & Solidago chilensis & koñí ßiar'á (flor amarilla) & $\mathrm{DE}$ \\
\hline
\end{tabular}




\section{G. F. Scarpa y C. N. Rosso - La etnobotánica moqoit inédita de Raúl Martínez Crovetto}

\begin{tabular}{|c|c|c|c|}
\hline Especie actual & Especie M.C. (lit.) & Fitónimo moqoit (castellano) & Tipo \\
\hline Sonchus asper (L.) Hill & Sonchus asper & palor'ó letaá (padre del cardo) & NP2 \\
\hline $\begin{array}{l}\text { Symphyotrichum squamatum } \\
\text { (Spreng.) G.L. Nesom }\end{array}$ & Aster squamatus & pewé nawerelék (yuyo que ennegrece) & NP2 \\
\hline Tagetes minuta L. & Tagetes minuta & $\begin{array}{l}\text { piók' nkor'onak'jlá' (donde orina } \\
\text { el perro); rer'ón (catinga) }\end{array}$ & $\begin{array}{l}\mathrm{DE} \\
\mathrm{DE}\end{array}$ \\
\hline $\begin{array}{l}\text { Tessaria dodoneifolia (Hook. \& } \\
\text { Arn.) Cabrera ssp. dodoneifolia }\end{array}$ & Tessaria dodonaefolia & noßogué / nowogué & NP \\
\hline Tessaria integrifolia Ruiz \& Pav. & Tessaria integrifolia & noßogué / nowogué & NP \\
\hline Trixis sp. & Trixis sp. (M681) & chimmak'-raík (picante) & $\mathrm{DE}$ \\
\hline $\begin{array}{l}\text { Vernonanthura chamaedrys } \\
\text { (Less.) H. Rob. }\end{array}$ & Vernonia chamaedrys & noßogué / nowogué & NP \\
\hline Vernonia sororia DC. & Vernonia scorpioides & lalar'aík (hoja blanca) & DE \\
\hline Vernonia sp. & Vernonia sp. (M679) & nawerelék (negruzco) & DE \\
\hline Vernonia sp. & Vernonia sp. (M682) & piók' latarík (remedio del perro) & DE \\
\hline Vernonia sp. & Vernonia sp. (M698) & noomr'á & $?$ \\
\hline Xanthium cavanillesii Schouw & Xanthium cavanillesii & ronái- olé (que se adhiere) & DE \\
\hline Xanthium spinosum L. var. spinosum & Xanthium spinosum & $\begin{array}{l}\text { ronái-olé lateé (la madre de los } \\
\text { que se clavan o adhieren); taasót } \\
\text { lateé (madre del cadillo) }\end{array}$ & $\begin{array}{l}\text { DE } \\
\text { NP2 }\end{array}$ \\
\hline \multicolumn{4}{|l|}{ AZOLLACEAE } \\
\hline Azolla filiculoides Lam. & Azolla filiculoides & k'ol & NP \\
\hline \multicolumn{4}{|l|}{ BASELLACEAE } \\
\hline cf. Anredera cordifolia (Ten.) Steenis & Borreria baselloides & nowelá & NP \\
\hline \multicolumn{4}{|l|}{ BIGNONIACEAE } \\
\hline $\begin{array}{l}\text { Amphilophium carolinae } \\
\text { (Lindl.) L. G. Lohmann }\end{array}$ & $\begin{array}{l}\text { Pithecoctenium } \\
\text { cynanchoides }\end{array}$ & nowelá & NP \\
\hline Dolichandra cynanchoides Cham. & $\begin{array}{l}\text { Dolichandra } \\
\text { cynanchoides }\end{array}$ & regatolëk lenát (Uña de gatito) & DE \\
\hline $\begin{array}{l}\text { Dolichandra unguis-cati } \\
\text { (L.) L.G. Lohmann }\end{array}$ & Doxantha unguis-cati & regatolëk lenát (Uña de gatito) & DE \\
\hline $\begin{array}{l}\text { Fridericia dichotoma } \\
\text { (Jacq.) L.G. Lohmann }\end{array}$ & Arrabidaea rhodantha & nowelá & NP \\
\hline $\begin{array}{l}\text { Handroanthus impetiginosus } \\
\text { (Mart. ex DC.) Mattos }\end{array}$ & Tabebuia ipe & laj-laí, laj-laité (casa del mosquito) & DE \\
\hline Tabebuia nodosa (Griseb.) Griseb. & Tabebuia nodosa & nechiník & NP \\
\hline \multicolumn{4}{|l|}{ BOMBACACEAE } \\
\hline Ceiba chodatii (Hassl.) Ravenna & Chorisia insignis & k'añík & NP \\
\hline $\begin{array}{l}\text { Ceiba speciosa (A. St.-Hil., A. } \\
\text { Juss. \& Cambess.) Ravenna }\end{array}$ & Chorisia speciosa & k'añík & NP \\
\hline \multicolumn{4}{|l|}{ BORAGINACEAE } \\
\hline $\begin{array}{l}\text { Cordia americana (L.) } \\
\text { Gottschling \& J.S. Mill. }\end{array}$ & Patagonula americana & dawák, wawák & NP \\
\hline Tournefortia salzmannii DC. & Tournefortia salzmannii & tok tr'áik & $?$ \\
\hline \multicolumn{4}{|l|}{ BRASSICACEAE } \\
\hline Brassica nigra (L.) W.D.J. Koch & Brassica nigra & nawerelék (negruzco) & $\mathrm{DE}$ \\
\hline Lepidium didymum L. & Coronopus didymus & nigsák nemaík (amuleto del zorrino) & DE \\
\hline Lepidium sp. & Lepidium sp. & nanáik nemaík (amuleto de la yarará) & DE \\
\hline \multicolumn{4}{|l|}{ BROMELIACEAE } \\
\hline Aechmea distichantha Lem. & Aechmea distichantha & pak'ál & NP \\
\hline Bromelia serra Griseb. & Bromelia serra & kotagué & NP \\
\hline Dyckia ferox Mez & Dickia ferox & k'otá' & NP \\
\hline
\end{tabular}




\begin{tabular}{|c|c|c|c|}
\hline Especie actual & Especie M.C. (lit.) & Fitónimo moqoit (castellano) & Tipo \\
\hline Dyckia ragonesei A. Cast. & Dickia ragonesei & k'otá' & NP \\
\hline Dyckia sp. & Dickia sp. & k'otá' & NP \\
\hline $\begin{array}{l}\text { Tillandsia aeranthos (Loisel.) } \\
\text { L.B. Sm. var. aeranthos }\end{array}$ & Tillandsia aeranthos & rapirá lakchí (las siete cabrillas?) & $\mathrm{DE}$ \\
\hline Tillandsia bandensis Baker & Tillandsia bandensis & rapirá lakchí (las siete cabrillas?) & $\mathrm{DE}$ \\
\hline Tillandsia capillaris Ruiz \& Pav. & Tillandsia hieronymi & $\begin{array}{l}\text { rapirá lakchí letaá (la madre } \\
\text { de las siete cabrillas?) }\end{array}$ & $\mathrm{DE}$ \\
\hline Tillandsia Ioliacea Mart. ex Schult. f. & Tillandsia Ioliacea & $\begin{array}{l}\text { rapirá lakchí letaá (la madre } \\
\text { de las siete cabrillas?) }\end{array}$ & $\mathrm{DE}$ \\
\hline Tillandsia lorentziana Griseb. & Tillandsia lorentziana & rapirá lakchí (las siete cabrillas?) & $\mathrm{DE}$ \\
\hline Tillandsia meridionalis Baker & Tillandsia meridionalis & rapirá lakchí (las siete cabrillas?) & $\mathrm{DE}$ \\
\hline Tillandsia reichenbachii Baker & Tillandsia tucumanensis & rapirá lakchí (las siete cabrillas?) & DE \\
\hline Tillandsia tricholepis Baker & Tillandsia tricholepis & rapirá lakchí (las siete cabrillas?) & $\mathrm{DE}$ \\
\hline \multicolumn{4}{|l|}{ BUDDLEJACEAE } \\
\hline $\begin{array}{l}\text { Buddleja stachyoides } \\
\text { Cham. \& Schltdl. }\end{array}$ & Buddleja brasiliensis & $\begin{array}{l}\text { llimak'ñichí lokolák (donde come el } \\
\text { picaflor); pelé nemaík (amuleto del ciervo) }\end{array}$ & $\mathrm{DE}$ \\
\hline \multicolumn{4}{|l|}{ CACTACEAE } \\
\hline Cereus argentinensis Britton \& Rose & Cereus argentinensis & notor'ok'ishík & NP \\
\hline Cereus forbesii Otto ex C.F. Först. & Cereus validus & leketr'áik & NP \\
\hline Cereus uruguayanus R. Kiesling & Cereus peruvianus & leketr'áik & NP \\
\hline Cereus sp. & Cereus sp. $\left(n^{\circ} 90\right)$ & leketr'áik lateé (madre de leketGáik) & NP2 \\
\hline Cereus sp. & Cereus sp. & notor'o k'ishík letaá (padre de notor'o k'ishik) & NP2 \\
\hline Cleistocactus baumannii (Lem.) Lem. & $\begin{array}{l}\text { Cleistocactus } \\
\text { baummannii }\end{array}$ & shigotói & NP \\
\hline Echinopsis sp. & Echinopsis sp. & neñigui llík & $?$ \\
\hline Harrisia martinii (Labour.) Britton & Harrisia martinii & yepát & NP \\
\hline Harrisia sp. & Harrisia sp. $\left(n^{\circ} 92\right)$ & notor'ok'ishík & NP \\
\hline $\begin{array}{l}\text { Opuntia anacantha Speg. var. } \\
\text { retrorsa (Speg.) R. Kiesling }\end{array}$ & Opuntia retrorsa & dak'-rák lok'oík (donde come la chuña) & DE \\
\hline $\begin{array}{l}\text { Opuntia elata Salm-Dyck var. } \\
\text { cardiosperma (K. Schum.) R. Kiesling }\end{array}$ & Opuntia chakensis & raiamík & NP \\
\hline Opuntia ficus-indica (L.) Mill.* & Opuntia vulgaris & raiamík & NP \\
\hline Opuntia ficus-indica (L.) Mill.* & Opuntia ficus-indica & ladók Ik'osót (cogote largo) & $\mathrm{DE}$ \\
\hline Opuntia ficus-indica (L.) Mill.* & Opuntia sp. $\left(n^{\circ} 81\right)$ & togué' (colorada) & $\mathrm{DE}$ \\
\hline Opuntia quimilo K. Schum. & Opuntia quimilo & shipr'ák' lolá('ík) (testículo de caballo) & $\mathrm{DE}$ \\
\hline Opuntia salmiana Parm. & Opuntia salmiana & redarák lokó (comida de la tortuga) & $\mathrm{DE}$ \\
\hline Opuntia sp. & Opuntia sp. $\left(n^{\circ} 64\right)$ & pishñí & $?$ \\
\hline Opuntia sp. & Opuntia sp. $\left(n^{\circ} 59\right)$ & toguiguí / toguiík (colorado por dentro) & $\mathrm{DE}$ \\
\hline Opuntia sp. & Opuntia sp. $\left(n^{\circ} 82\right)$ & tor'oík (colorado por dentro) & $\mathrm{DE}$ \\
\hline Opuntia sp. & Opuntia sp. $\left(n^{\circ} 69\right)$ & relank'a e lete' & $?$ \\
\hline Opuntia sp. & Opuntia sp. $\left(n^{\circ} 41\right)$ & konor'óik & $?$ \\
\hline Rhipsalis sp. & Rhipsalis sp. & $\begin{array}{l}\text { k'onasé laañík (soga de la } \\
\text { mujer duende del monte) }\end{array}$ & DE \\
\hline $\begin{array}{l}\text { Stetsonia coryne (Salm- } \\
\text { Dyck) Britton \& Rose }\end{array}$ & Cereus coryne & leketr'áik & NP \\
\hline \multicolumn{4}{|l|}{ CALYCERACEAE } \\
\hline Acicarpha tribuloides Juss. & Acicarpha tribuloides & palor'ó lateé (madre del cardo) & NP2 \\
\hline \multicolumn{4}{|l|}{ CANNACEAE } \\
\hline Canna glauca L. & Canna glauca & pe'lák & NP \\
\hline Canna indica L. & Canna indica & pe'lák & NP \\
\hline \multicolumn{4}{|l|}{ CAPPARACEAE } \\
\hline $\begin{array}{l}\text { Anisocapparis speciosa (Griseb.) } \\
\text { X. Cornejo \& H.H. Iltis }\end{array}$ & Capparis speciosa & nelamík & NP \\
\hline
\end{tabular}


G. F. Scarpa y C. N. Rosso - La etnobotánica moqoit inédita de Raúl Martínez Crovetto

\begin{tabular}{|c|c|c|c|}
\hline Especie actual & Especie M.C. (lit.) & Fitónimo moqoit (castellano) & Tipo \\
\hline $\begin{array}{l}\text { Capparicordis tweediana (Eichler) } \\
\text { H.H. Iltis \& X. Cornejo }\end{array}$ & Capparis tweediana & kiyillík; kiyilligué' & NP \\
\hline Capparis atamisquea Kuntze & Atamisquea emarginata & lak'aßiar'aík (quebradizo) & DE \\
\hline Capparis flexuosa (L.) L. & $\begin{array}{l}\text { Capparis } \\
\text { cynophallophora }\end{array}$ & neteguék & $\begin{array}{l}N P \\
N P\end{array}$ \\
\hline $\begin{array}{l}\text { Cynophalla retusa (Griseb.) } \\
\text { X. Cornejo \& H.H. Iltis }\end{array}$ & Capparis retusa & neteguék & $\begin{array}{l}\text { NP } \\
\text { NP }\end{array}$ \\
\hline \multicolumn{4}{|l|}{ CARICACEAE } \\
\hline Carica quercifolia (A. St.-Hil.) Hieron. & Carica quercifolia & nakalmaík & NP \\
\hline CARYOPHYLLACEAE & Caryophyllaceae (M702) & pewé lachík & NP2 \\
\hline \multicolumn{4}{|l|}{ CELASTRACEAE } \\
\hline cf. Maytenus vitis-idaea Griseb. & (M671) & satachík (lo come + ?) & DE \\
\hline Moya spinosa Griseb. & Maytenus spinosa & tok leí (líquido colorado) & DE \\
\hline \multicolumn{4}{|l|}{ CELTIDACEAE } \\
\hline Celtis chichape (Wedd.) Miq. & Celtis pubescens & penkoshík & NP \\
\hline Celtis iguanaea (Jacq.) Sarg. & Celtis spinosa & penkoshík & NP \\
\hline Celtis iguanaea (Jacq.) Sarg. & Celtis iguanea & penkoshík & NP \\
\hline Celtis sp. & Celtis sp. (M684) & penkoshík lateé & NP2 \\
\hline \multicolumn{4}{|l|}{ CERVANTESIACEAE } \\
\hline $\begin{array}{l}\text { Jodina rhombifolia (Hook. } \\
\text { \& Arn.) Reissek }\end{array}$ & Jodina rhombifolia & shichí' labík & $?$ \\
\hline \multicolumn{4}{|l|}{ CHENOPODIACEAE } \\
\hline $\begin{array}{l}\text { Dysphania ambrosioides (L.) } \\
\text { Mosyakin \& Clemants }\end{array}$ & $\begin{array}{l}\text { Chenopodium } \\
\text { ambrosioides }\end{array}$ & rebaullí letaá (padre del rebaullí) & NP2 \\
\hline Sarcocornia sp. & Salicornia sp. & hoo-wé (sal) & $\mathrm{DE}$ \\
\hline \multicolumn{4}{|l|}{ COMMELINACEAE } \\
\hline Commelina erecta L. & Commelina erecta & $\begin{array}{l}\text { k'agueretá la/oné (vulva del guazuncho); } \\
\text { k'amí latá (remedio para dolor de vista) }\end{array}$ & $\begin{array}{l}\mathrm{DE} \\
\mathrm{DE}\end{array}$ \\
\hline \multicolumn{4}{|l|}{ CONVOLVULACEAE } \\
\hline Convolvulus crenatifolius Ruiz \& Pav. & Convolvulus crenatifolius & nowelá & NP \\
\hline Convolvulus hermanniae L'Hér. & Convolvulus hermanniae & nowelá & NP \\
\hline $\begin{array}{l}\text { Dichondra microcalyx } \\
\text { (Hallier f.) Fabris }\end{array}$ & Dichondra repens & $\begin{array}{l}\text { nesor'oná nemaík (amuleto o payé } \\
\text { del "apereá") (Cavia aperea) }\end{array}$ & DE \\
\hline Ipomoea amnicola Morong & Ipomoea amnicola & nowelá & NP \\
\hline $\begin{array}{l}\text { Ipomoea bonariensis Hook } \\
\text { var. bonariensis }\end{array}$ & $\begin{array}{l}\text { Ipomoea bonariensis } \\
\text { var. chacoensis }\end{array}$ & saalrá & NP \\
\hline Ipomoea cairica (L.) Sweet & Ipomoea cairica & nowelá & NP \\
\hline $\begin{array}{l}\text { Ipomoea carnea Jacq. ssp. fistulosa } \\
\text { (Mart. ex Choisy) D.F. Austin }\end{array}$ & Ipomoea fistulosa & kané newomá (cántaro que se rompe) & DE \\
\hline Ipomoea indica (Burm. f.) Merr & Ipomoea congesta & nowelá & NP \\
\hline Ipomoea nil (L.) Roth & Ipomoea nil & kanr'ái newomá (cántaro roto), nowelá & $\begin{array}{l}\text { DE } \\
\text { NP }\end{array}$ \\
\hline \multicolumn{4}{|l|}{ CUCURBITACEAE } \\
\hline $\begin{array}{l}\text { Cayaponia citrullifolia (Griseb.) } \\
\text { Cogn. ex Griseb. }\end{array}$ & Cayaponia citrullifolia & nanáik lokó (comida de la yarará) & $\mathrm{DE}$ \\
\hline Cayaponia podantha Cogn. & Cayaponia podantha & nelomák' nemaík (amuleto de la cascabel) & $\mathrm{DE}$ \\
\hline $\begin{array}{l}\text { Citrullus lanatus (Thunb.) Matsum. } \\
\text { \& Nakai ssp. vulgaris (Schrad. } \\
\text { ex Eckl. \& Zeyh.) Fursa** }\end{array}$ & Citrullus vulgaris & neßagué laße (hoja ¿?) & $\mathrm{DE}$ \\
\hline Cucumis melo L.* & Cucumis melo & chik-rar'ái (azul ¿?) & DE \\
\hline Cucurbita maxima Duchesne * & Cucurbita maxima & log-guillí & NP \\
\hline Cucurbita pepo L.* & Cucurbita pepo & log-guillí & NP \\
\hline
\end{tabular}




\begin{tabular}{|c|c|c|c|}
\hline Especie actual & Especie M.C. (lit.) & Fitónimo moqoit (castellano) & Tipo \\
\hline $\begin{array}{l}\text { Cucurbitella asperata (Gillies } \\
\text { ex Hook. \& Arn.) Walp. }\end{array}$ & Cucurbitella duriaei & nanáik lokó (comida de la yarará) & $\mathrm{DE}$ \\
\hline Lagenaria siceraria (Molina) Standl. * & Lagenaria siceraria & k'apar'á & NP \\
\hline Melothria cucumis Vell. & Melothria cucumis & $\begin{array}{l}\text { k'agueretá la/oeté (teta del guazuncho); } \\
\text { nanáik lokó (comida de la yarará) }\end{array}$ & $\begin{array}{l}\mathrm{DE} \\
\mathrm{DE}\end{array}$ \\
\hline \multicolumn{4}{|l|}{ CYPERACEAE } \\
\hline Bulbostylis capillaris (L.) C.B. Clarke & Bulbostylis capillaris & waká lók (comida de la vaca) & DE \\
\hline $\begin{array}{l}\text { Bulbostylis juncoides (Vahl) } \\
\text { Kük. ex Herter var. lorentzii } \\
\text { (Boeck.) Kük. ex Osten }\end{array}$ & Bulbostylis juncoides & retañí & NP \\
\hline Cyperus digitatus Roxb. & Cyperus digitatus & retañí & NP \\
\hline Cyperus entrerianus Boeck. & Cyperus entrerianus & retañí & NP \\
\hline Cyperus giganteus Vahl & Cyperus giganteus & rapiar'rán & NP \\
\hline Cyperus luzulae (L.) Rottb. ex Retz. & Cyperus luzulae & retañí & NP \\
\hline Cyperus odoratus L. & Cyperus ferax & retañí & NP \\
\hline Cyperus sp. & Cyperus sp. (M700) & rapiar'rán; piar'rán & NP \\
\hline Cyperus sp. & Cyperus sp. (M701) & rapiar'rán letaá & NP2 \\
\hline Cyperus sp. & Cyperus sp. (M716) & rapiar'rán & NP \\
\hline $\begin{array}{l}\text { Eleocharis montana (Kunth) } \\
\text { Roem. \& Schult. }\end{array}$ & Eleocharis nodulosa & rachipiolé (reventoncito) & $\mathrm{DE}$ \\
\hline Eleocharis sp. & Eleocharis sp. (M670) & rachipiolé (reventoncito) & DE \\
\hline Rhynchospora corymbosa (L.) Britton & $\begin{array}{l}\text { Rhynchospora } \\
\text { corymbosa }\end{array}$ & rapiar'rán & NP \\
\hline $\begin{array}{l}\text { Schoenoplectus californicus } \\
\text { (C.A. Mey.) Soják }\end{array}$ & Scirpus californicus & naßaté & NP \\
\hline \multicolumn{4}{|l|}{ DIOSCOREACEAE } \\
\hline Dioscorea sp. & Dioscorea brachybothria & wák-iák leté (aguachenta) & DE \\
\hline \multicolumn{4}{|l|}{ EPHEDRACEAE } \\
\hline Ephedra sp. & Ephedra sp. & k'oteták' & NP \\
\hline $\begin{array}{l}\text { Ephedra triandra Tul. } \\
\text { emend. J.H. Hunz. }\end{array}$ & Ephedra triandra & k'oteták' letaá (padre del k'oteták') & NP2 \\
\hline \multicolumn{4}{|l|}{ EQUISETACEAE } \\
\hline Equisetum giganteum $\mathrm{L}$. & Equisetum giganteum & k'oteták'; shiprák' Ik'aiít (cola de caballo) & PRE \\
\hline \multicolumn{4}{|l|}{ ERYTHROXYLACEAE } \\
\hline Erythroxylum argentinum O.E. Schulz & Erythroxylon argentinum & k'ochiñí lokó' (comida de la charata) & DE \\
\hline \multicolumn{4}{|c|}{ EUPHORBIACEAE } \\
\hline Acalypha communis Müll. Arg. & Acalypha guaranitica & pewé (yuyo) & NP \\
\hline Acalypha polystachya Jacq. * & Acalypha polystachya & pewé (yuyo) & NP \\
\hline Croton argenteus L. & Julocroton montevidense & migran (?) & $?$ \\
\hline Croton lachnostachyus Baill. & $\begin{array}{l}\text { Croton aff. } \\
\text { lachnostachyus }\end{array}$ & toguiguí; togui ík (colorado por dentro) & DE \\
\hline Euphorbia serpens Kunth & Euphorbia serpens & repálguiót nemaík (amuleto de la golondrina) & PRE \\
\hline Manihot esculenta Crantz & Manihot dulcis & manyóka & PRE \\
\hline Manihot esculenta Crantz & Manihot utilissima & manyóka & PRE \\
\hline Ricinus communis L. & Ricinus communis & redonatr'án & NP \\
\hline Sapium haematospermum Müll. Arg. & $\begin{array}{l}\text { Sapium } \\
\text { haematospermum }\end{array}$ & ro'ík & NP \\
\hline Tragia geraniifolia Klotzsch ex Baill. & Tragia geraniifolia & pok'oík (que quema) & DE \\
\hline Tragia volubilis $\mathrm{L}$ & Tragia volubilis & pok'oík (que quema) & DE \\
\hline \multicolumn{4}{|l|}{ FABACEAE } \\
\hline Acacia aroma Gillies ex Hook.\& Arn. & Acacia aroma & pak'-kik & NP \\
\hline Acacia atramentaria Benth. & Acacia atramentaria & uak'aík & NP \\
\hline
\end{tabular}




\section{G. F. Scarpa y C. N. Rosso - La etnobotánica moqoit inédita de Raúl Martínez Crovetto}

\begin{tabular}{|c|c|c|c|}
\hline Especie actual & Especie M.C. (lit.) & Fitónimo moqoit (castellano) & Tipo \\
\hline $\begin{array}{l}\text { Acacia bonariensis Gillies } \\
\text { ex Hook. \& Arn. }\end{array}$ & Acacia bonariensis & uak'aík & \\
\hline Acacia caven (Molina) Molina & Acacia caven & pak'-kik & NP \\
\hline Acacia praecox Griseb. & Acacia praecox & uak'aík & NP \\
\hline Aeschynomene rudis Benth. & Aeschynomene rudis & pok'ok raík letaá (padre de la vergonzosa) & $\mathrm{DE}$ \\
\hline $\begin{array}{l}\text { Arachis glabrata Benth. } \\
\text { var. hagenbeckii (Harms } \\
\text { ex Kuntze) F.J. Herm. }\end{array}$ & Arachis hagenbeckii & waká nemaík (amuleto de la vaca) & $\mathrm{DE}$ \\
\hline Arachis hypogaea L.* & Arachis hypogaea & mañí & PRE \\
\hline $\begin{array}{l}\text { Bauhinia forficata Link ssp. pruinosa } \\
\text { (Vogel) Fortunato \& Wunderlin }\end{array}$ & Bahuinia candicans & waká lapiá' (pata de vaca) & PRE \\
\hline $\begin{array}{l}\text { Caesalpinia paraguariensis } \\
\text { (D. Parodi) Burkart }\end{array}$ & $\begin{array}{l}\text { Caesalpinia } \\
\text { paraguariensis }\end{array}$ & nallikaík & NP \\
\hline Canavalia ensiformis (L.) DC. & Canavalia ensiformis & nabirichiguí (la que se arrastra) & DE \\
\hline $\begin{array}{l}\text { Desmanthus tatuhyensis Hoehne } \\
\text { var. brevipes (B.L. Turner) Luckow }\end{array}$ & Desmanthus chacoense & pok'ó raík (que tiene vergüenza) & $\mathrm{DE}$ \\
\hline Desmodium cuneatum Hook. \& Arn. & Desmodium cuneatum & shiprák' lamaná (que le gusta al caballo) & DE \\
\hline Desmodium incanum DC. & Desmodium canum & maañík lok (comida del suri) & $\mathrm{DE}$ \\
\hline $\begin{array}{l}\text { Enterolobium contortisiliquum } \\
\text { (Vell.) Morong }\end{array}$ & $\begin{array}{l}\text { Enterolobium } \\
\text { contortisiliquum (M712) }\end{array}$ & telraík; tebiaraík & NP \\
\hline Erythrina crista-galli L. var. crista-galli & Erythrina crista-galli & nechiník & NP \\
\hline $\begin{array}{l}\text { Geoffroea decorticans (Gillies } \\
\text { ex Hook. \& Arn.) Burkart }\end{array}$ & Geoffroea decorticans & petekaík & NP \\
\hline $\begin{array}{l}\text { Gleditsia amorphoides (Griseb.) } \\
\text { Taub. var. amorphoides }\end{array}$ & Gleditsia amorphoides & nowishík & NP \\
\hline Indigofera sp. & Indigofera sp. (M695) & rerón olé (catinguita) & DE \\
\hline Medicago sativa $\mathrm{L}$. & Medicago sativa & álpa & PRE \\
\hline Mimosa detinens Benth. & Mimosa detinens & netetr'án k'achík & $?$ \\
\hline Mimosa strigillosa Torr. \& A. Gray & Mimosa strigillosa & pok'ó r'aík (que tiene vergüenza) & $\mathrm{DE}$ \\
\hline Parkinsonia aculeata L. & Parkinsonia aculeata & neßishkík & NP \\
\hline Poiretia tetraphylla (Poir.) Burkart & Poiretia tetraphylla & sar'á Ipinék (hueso ¿?) & $\mathrm{DE}$ \\
\hline Prosopis affinis Spreng. & Prosopis algarrobilla & pikñík & NP \\
\hline Prosopis alba Griseb. & Prosopis alba & maapík & NP \\
\hline Prosopis kuntzei Harms & Prosopis kuntzei & netarguék & NP \\
\hline Prosopis nigra (Griseb.) Hieron. & Prosopis nigra & naßeték lelkáik & $\mathrm{DE}$ \\
\hline Prosopis ruscifolia Griseb. & Prosopis ruscifolia & (ne)ñirashík & $?$ \\
\hline $\begin{array}{l}\text { Prosopis sericantha Gillies } \\
\text { ex Hook. \& Arn. }\end{array}$ & Prosopis sericantha & netarguék lateé (madre del netarguék) & NP2 \\
\hline Rhynchosia diversifolia Micheli & Rhynchosia diversifolia & koñiguí (amarilla ¿?) & $\mathrm{DE}$ \\
\hline $\begin{array}{l}\text { Senna corymbosa (Lam.) } \\
\text { H.S. Irwin \& Barneby }\end{array}$ & Cassia corymbosa & $\begin{array}{l}\text { nowaiar'á namáp / nowar'air'á } \\
\text { namáp (vaina del zorro / aguará) }\end{array}$ & $\mathrm{DE}$ \\
\hline $\begin{array}{l}\text { Senna hirsuta (L.) H.S. Irwin } \\
\text { \& Barneby var. leptocarpa } \\
\text { (Benth.) H.S. Irwin \& Barneby }\end{array}$ & Cassia leptocarpa & $\begin{array}{l}\text { nowaiar'á namáp / nowar'air'á } \\
\text { namáp (vaina del zorro / aguará) }\end{array}$ & DE \\
\hline $\begin{array}{l}\text { Senna morongii (Britton) } \\
\text { H.S. Irwin \& Barneby }\end{array}$ & Cassia morongii & $\begin{array}{l}\text { nowaiar'á namáp / nowar'air'á } \\
\text { namáp (vaina del zorro / aguará) }\end{array}$ & $\mathrm{DE}$ \\
\hline Senna occidentalis (L.) Link & Cassia occidentalis & $\begin{array}{l}\text { nowaiar'á namáp / nowar'air'á } \\
\text { namáp (vaina del zorro / aguará) }\end{array}$ & DE \\
\hline $\begin{array}{l}\text { Senna rigida (Hieron.) } \\
\text { H.S. Irwin \& Barneby }\end{array}$ & Cassia rigida & netarguék letaá (padre del netarguék) & NP2 \\
\hline $\begin{array}{l}\text { Senna pendula (Willd.) H.S. } \\
\text { Irwin \& Barneby var. paludicola } \\
\text { H.S. Irwin \& Barneby }\end{array}$ & Cassia bicapsularis & $\begin{array}{l}\text { nowaiar'á namáp / nowar'air'á } \\
\text { namáp (vaina del zorro / aguará) }\end{array}$ & $\mathrm{DE}$ \\
\hline
\end{tabular}


Bol. Soc. Argent. Bot. 49 (4) 2014

\begin{tabular}{|c|c|c|c|}
\hline Especie actual & Especie M.C. (lit.) & Fitónimo moqoit (castellano) & Tipo \\
\hline LAMIACEAE & Lamiaceae (M693) & koñiguí letaá (madre de la raíz amarilla) & \\
\hline Hyptis lappacea Benth & Hyptis lappacea & $\begin{array}{l}\text { nak'atek Ikolák (donde chupa la } \\
\text { lechiguana); naweté namak'á lkolák } \\
\text { (donde chupa el abejorro) }\end{array}$ & $\begin{array}{l}\mathrm{DE} \\
\mathrm{DE}\end{array}$ \\
\hline Leonurus cardiaca L. & Leonorus cardiaca & pewé (yuyo) & NP \\
\hline Salvia cardiophylla Benth. & Salvia cardiophylla & $\begin{array}{l}\text { señít halók (comida del peludo); } \\
\text { ik'olák le'noßiar'ái (flor azul) }\end{array}$ & $\begin{array}{l}\mathrm{DE} \\
\mathrm{DE}\end{array}$ \\
\hline \multicolumn{4}{|l|}{ LEMNACEAE } \\
\hline Lemna gibba L. & Lemna gibba & k'ol / re-topí & $\begin{array}{l}\text { NP; } \\
?\end{array}$ \\
\hline \multicolumn{4}{|l|}{ LILIACEAE } \\
\hline Allium cepa L.* & Allium cepa & k'alachí & NP \\
\hline Allium sativum $\mathrm{L} .{ }^{*}$ & Allium sativum & ak'ós & PRE \\
\hline \multicolumn{4}{|l|}{ LORANTHACEAE } \\
\hline $\begin{array}{c}\text { Ligaria cuneifolia (Ruiz \& Pav.) Tiegh. } \\
\text { LYTHRACEAE }\end{array}$ & Psittacanthus cuneifolius & k(')ak(')aré lkaík (cabeza de carancho) & DE \\
\hline Heimia salicifolia (Kunth) Link & Heimia salicifolia & $\begin{array}{l}\text { paten nk'achík / pate(n)rank'achík } \\
\text { (para estaquear); sará Ipinék }\end{array}$ & $\begin{array}{l}\mathrm{DE} \\
?\end{array}$ \\
\hline \multicolumn{4}{|l|}{ MALPIGHIACEAE } \\
\hline $\begin{array}{l}\text { Janusia guaranitica (A. } \\
\text { St.-Hil.) A. Juss. }\end{array}$ & Janusia guaranitica & nowelá & NP \\
\hline MALVACEAE & Malvaceae (M661) & $\begin{array}{l}\text { koñilalá nemaík letaá (madre } \\
\text { del amuleto de la rubita) }\end{array}$ & $\mathrm{DE}$ \\
\hline Abutilon sp. & Abutilon sp. & $\begin{array}{l}\text { señít halók letaá (madre de } \\
\text { la comida del peludo) }\end{array}$ & DE \\
\hline Cienfuegosia sp. & Cienfuegosia sp. (M707) & koñíle nowiar'ái-olé (florcita amarilla) & $\mathrm{DE}$ \\
\hline Gossypium sp. & Gossypium herbaceum? & ualók & NP \\
\hline Hibiscus striatus Cav. & Hibiscus lambertianus & kanr'ái newomá (cántaro roto) & $\mathrm{DE}$ \\
\hline Hibiscus striatus Cav. & Hibiscus cisplatinus & kanr'ái newomá (cántaro roto) & DE \\
\hline $\begin{array}{l}\text { Hochreutinera hasslerana } \\
\text { (Hochr.) Krapov }\end{array}$ & Abutilon hasslerianum & $\begin{array}{l}\text { señít halók letaá (madre de } \\
\text { la comida del peludo) }\end{array}$ & DE \\
\hline $\begin{array}{l}\text { Malvastrum coromandelianum } \\
\text { (L.) Garcke }\end{array}$ & $\begin{array}{l}\text { Malvastrum } \\
\text { coromandelianum }\end{array}$ & naráik peßé (yuyo duro, yuyo) peßé (yuyo) & $\begin{array}{l}\text { NP2 } \\
\text { NP }\end{array}$ \\
\hline Sida rhombifolia L. & Sida rhombifolia & pewé (yuyo) & NP \\
\hline $\begin{array}{l}\text { Sphaeralcea bonariensis } \\
\text { (Cav.) Griseb. }\end{array}$ & Sphaeralcea bonariensis & señít halók (comida del peludo) & $\mathrm{DE}$ \\
\hline Wissadula densiflora R.E. Fr. & Wissadula densiflora & kesak'sé (que brota en el patio) & DE \\
\hline \multicolumn{4}{|l|}{ MARANTACEAE } \\
\hline Thalia geniculata L. & Thalia geniculata & $\begin{array}{l}\text { naanók lapotó (poncho del } \\
\text { yacaré) }\end{array}$ & $\begin{array}{l}\text { DE } \\
\text { NP }\end{array}$ \\
\hline Thalia multiflora Horkel & Thalia sp. & naanók lapotó (poncho del yacaré); k’añik & $\begin{array}{l}\text { DE } \\
\text { NP }\end{array}$ \\
\hline \multicolumn{4}{|l|}{ MARTYNIACEAE } \\
\hline Ibicella lutea (Lindl.) Van Eselt. & Ibicella lutea & naiiapëk lasoté (cuerno del diablo) & PRE \\
\hline \multicolumn{4}{|l|}{ MELIACEAE } \\
\hline Melia azedarach L.* & Melia azederach & paraík (de "paraíso) & PRE \\
\hline \multicolumn{4}{|l|}{ MORACEAE } \\
\hline Broussonettia papyrifera* (L.) Vent. & Brousonettia papyrifera & lalar'ráik lateé (planta de sombra) & DE \\
\hline Ficus carica L.* & Ficus carica & piók' laeté (teta del perro) & $\mathrm{DE}$ \\
\hline Ficus luschnathiana (Miq.) Miq. & Ficus monckii & shruík & NP \\
\hline $\begin{array}{l}\text { Maclura tinctoria (L.) } \\
\text { Steud. ssp. tinctoria }\end{array}$ & $\begin{array}{l}\text { Chlorophora tinctoria } \\
\text { var. xanhoxylon }\end{array}$ & nokoilák naté (resina violácea) & $\mathrm{DE}$ \\
\hline
\end{tabular}


G. F. Scarpa y C. N. Rosso - La etnobotánica moqoit inédita de Raúl Martínez Crovetto

\begin{tabular}{|c|c|c|c|}
\hline Especie actual & Especie M.C. (lit.) & Fitónimo moqoit (castellano) & Tipo \\
\hline \multicolumn{4}{|l|}{ MYRTACEAE } \\
\hline Eucalyptus camaldulensis Dehnh.* & Eucalyptus camaldulensis & doimi(a)r'ái & $?$ \\
\hline Eucalyptus tereticornis Sm.* & Eucalyptus tereticornis & doimi(a)r'ái & $?$ \\
\hline Eugenia uniflora L. & Eugenia uniflora & taik'ók & NP \\
\hline \multicolumn{4}{|l|}{ NYCTAGINACEAE } \\
\hline Boerhavia diffusa L. var. diffusa & Boerhavia paniculata & kos lok (comida del cerdo) & DE \\
\hline Pisonia zapallo Griseb. & Pisonia zapallo & nashiwík & NP \\
\hline \multicolumn{4}{|l|}{ NYMPHAEACEAE } \\
\hline Victoria cruziana Orb. & Victoria cruziana & naanók lapotó (poncho del yacaré) & DE \\
\hline \multicolumn{4}{|l|}{ ONAGRACEAE } \\
\hline $\begin{array}{l}\text { Ludwigia elegans } \\
\text { (Cambess.) H. Hara }\end{array}$ & Jussiaea elegans & koñí le'noßiar'ái (flor amarilla) & DE \\
\hline $\begin{array}{l}\text { Ludwigia peploides (Kunth) } \\
\text { P.H. Raven ssp. peploides }\end{array}$ & Jussiaea repens & $\begin{array}{l}\text { koñí le'noßiar'ái (flor amarilla) } \\
\text { tak'ák' lechí (pata de chajá) }\end{array}$ & $\begin{array}{l}\text { DE } \\
\text { NP2 }\end{array}$ \\
\hline \multicolumn{4}{|l|}{ ORCHIDACEAE } \\
\hline $\begin{array}{l}\text { cf. Sarcoglottis grandiflora } \\
\text { (Hook.) Klotzsch }\end{array}$ & Orchidaceae & pe'lák letaá (padre de la achira) & NP2 \\
\hline Cyclopogon sp. & Cyclopogon sp. & togué' le'noßiar'ái (flor colorada) & DE \\
\hline Oncidium bifolium Sims var. bifolium & Oncidium bifolium & shirík & NP \\
\hline \multicolumn{4}{|l|}{ OXALIDACEAE } \\
\hline Oxalis conorrhiza Jacq. & Oxalis cordobensis & $\begin{array}{l}\text { koñí le'noßiar'ái (flor amarilla) } \\
\text { koñí peße (yuyo amarillo) }\end{array}$ & $\begin{array}{l}\text { DE } \\
\text { NP2 }\end{array}$ \\
\hline \multicolumn{4}{|l|}{ PASSIFLORACEAE } \\
\hline Passiflora mooreana Hook. f. & Passiflora mooreana & piók' lola(r)ík (testículos de perro) & DE \\
\hline Passiflora sp. & Passiflora sp. & nanáik nemaík (amuleto de la yarará) & DE \\
\hline \multicolumn{4}{|l|}{ PHYTOLACCACEAE } \\
\hline Petiveria alliacea L. var. alliacea & Petiveria alliacea & shipotók & NP \\
\hline Phytolacca dioica L. & Phytolacca dioica & $\begin{array}{l}\text { nakalmaík lateé (madre del } \\
\text { mamón silvestre) }\end{array}$ & NP2 \\
\hline Rivina humilis L. & Rivina humilis & kaoó nkoté (ojo del pájaro guaycurú) & DE \\
\hline \multicolumn{4}{|l|}{ PIPERACEAE } \\
\hline $\begin{array}{l}\text { Peperomia comarapana } \\
\text { C. DC. f. comarapana }\end{array}$ & Peperomia comarapana & $\begin{array}{l}\text { ñiló' latá (remedio contra la } \\
\text { queresa -miasis-) }\end{array}$ & $\mathrm{DE}$ \\
\hline \multicolumn{4}{|l|}{ PLANTAGINACEAE } \\
\hline Plantago australis Lam. var. australis & Plantago macrostachys & lalar'ái lawé (hoja blanca) & DE \\
\hline Scoparia sp. & Scoparia sp. (M703) & koñiguí (raíz amarilla) & DE \\
\hline POACEAE & Gramínea (n79) & kepok'yak & $?$ \\
\hline Arundo donax L. & Arundo donax & $\begin{array}{l}\text { (no)k'olá lateé (madre de la } \\
\text { tacuara), k'olá lachinik }\end{array}$ & $\begin{array}{l}\text { NP2 } \\
\text { NP2 }\end{array}$ \\
\hline $\begin{array}{l}\text { Avena byzanthina K. Koch } \\
\text { biaristata (Hack. ex Trab.) Parodi }\end{array}$ & Avena byzanthina & lo'otá & NP \\
\hline $\begin{array}{l}\text { Bothriochloa exaristata } \\
\text { (Nash) Henrard }\end{array}$ & Bothriochloa hassleri & tok Ipinék (hueso rojo) & DE \\
\hline $\begin{array}{l}\text { Bothriochloa laguroides (DC.) } \\
\text { Herter var. laguroides }\end{array}$ & Bothriochloa laguroides & uak'apí & NP \\
\hline $\begin{array}{l}\text { Bothriochloa saccharoides (Sw.) } \\
\text { Rydb. var. saccharoides }\end{array}$ & $\begin{array}{l}\text { Bothriochloa } \\
\text { saccharoides }\end{array}$ & uak'apí & NP \\
\hline Cenchrus echinatus L. & Cenchrus echinatus & taasót & NP \\
\hline $\begin{array}{l}\text { Cenchrus longispinus } \\
\text { (Hack.) Fernald. }\end{array}$ & Cenchrus pauciflorus & taasót & NP \\
\hline Cenchrus myosuroides Kunth & Cenchrus myosuroides & taasót & NP \\
\hline
\end{tabular}




\begin{tabular}{|c|c|c|c|}
\hline Especie actual & Especie M.C. (lit.) & Fitónimo moqoit (castellano) & Tipo \\
\hline $\begin{array}{l}\text { Cenchrus pilcomayensis } \\
\text { (Mez) Morrone }\end{array}$ & Pennisetum frutescens & iém & NP \\
\hline Chloris ciliata Sw. & Chloris ciliata & uak'apí & NP \\
\hline Chloris sp. & Chloris sp. (M688) & lalagar'áik wak'apí (pasto blanco) & NP2 \\
\hline Coleataenia prionitis (Nees) Soreng & Panicum prionitis & rapiar'án & NP \\
\hline $\begin{array}{l}\text { Cortaderia selloana (Schult. \& } \\
\text { Schult. f.) Asch. \& Graebn. }\end{array}$ & Cortaderia selloana & palor'ó rachipiolé (reventoncito) & $\begin{array}{l}\text { NP } \\
\text { DE }\end{array}$ \\
\hline Cymbopogon citratus (DC.) Stapf & Cymbopogon citratus & lek'aliñí loiól lateé (madre de la mata ancha) & DE \\
\hline Cynodon dactylon L. & Cynodon dactylon & por'anr'áik & NP \\
\hline Digitaria insularis (L.) Fedde & Digitaria insularis & kolmáik & NP \\
\hline Digitaria sp. & Digitaria penniciligera & uak'apí & NP \\
\hline Elionurus lividus Hack. & Elionurus viridulus & chemmaGuá (amargo) & DE \\
\hline $\begin{array}{l}\text { Elionurus tripsacoides Humb. } \\
\text { \& Bonpl. ex Willd. }\end{array}$ & Elionurus tripsacoides & chemmaGuá (amargo) & DE \\
\hline Eragrostis airoides Nees & Eragrostis airoides & mañík radé (pestaña del suri), penék & $\begin{array}{l}\mathrm{DE} \\
\mathrm{NP}\end{array}$ \\
\hline $\begin{array}{l}\text { Eragrostis cilianensis (All.) } \\
\text { Vignolo ex Janch. }\end{array}$ & Eragrostis cilianensis & wak'apí letaá (padre del pasto) & NP2 \\
\hline Eragrostis lugens Nees & Eragrostis lugens & mañík radé (pestaña del suri) & DE \\
\hline Erianthus trinii (Hack.) Hack. & Erianthus trinii & rachipiolé (reventoncito) & $\mathrm{DE}$ \\
\hline Eriochloa montevidensis Griseb. & Eriochloa montevidensis & wak'apí letaá (padre del pasto) & NP2 \\
\hline $\begin{array}{l}\text { Guadua chacoensis (Rojas) } \\
\text { Londoño \& P.M. Peterson }\end{array}$ & Guadua angustifolia & (no)k'olá lateé (madre de la tacuara) & NP2 \\
\hline Guadua paraguayana Döll & Guadua paraguayana & k'olá & NP \\
\hline Leptochloa virgata (L.) P. Beauv. & Leptochloa virgata & penék & NP \\
\hline Melica macra Nees & Melica macra & shit & NP \\
\hline Oryza sativa L. & Oryza sativa & r'ro & PRE \\
\hline Panicum bergii Arechav. var. bergii & Panicum bergii & penék & NP \\
\hline $\begin{array}{l}\text { Pappophorum pappiferum } \\
\text { (Lam.) Kuntze }\end{array}$ & $\begin{array}{l}\text { Pappophorum } \\
\text { pappiferum }\end{array}$ & komdái & NP \\
\hline Paspalum coryphaeum Trin. & Paspalum brunneum & lek'aliñí loiól (mata ancha o sebácea) & DE \\
\hline $\begin{array}{l}\text { Paspalum notatum } \\
\text { Flüggé var. notatum }\end{array}$ & Paspalum notatum & ka'alañí letaá (padre del aplastado) & DE \\
\hline Paspalum plicatulum Michx. & Paspalum plicatulum & wak'apí lekálk (pasto grande) & NP2 \\
\hline Paspalum urvillei Steud. & Paspalum urvillei & uak'apí & NP \\
\hline Pharus lappulaceus Aubl. & Pharus glaber & ronái- olé (que se adhiere) & $\mathrm{DE}$ \\
\hline $\begin{array}{l}\text { Phyllostachys nigra (Lodd. } \\
\text { ex Lindl.) Munro }\end{array}$ & Phyllostachys nigra & k'olá & NP \\
\hline $\begin{array}{l}\text { Schizachyrium microstachyum (Desv. } \\
\text { ex Ham.) Roseng., B.R. Arrill. \& Izag. }\end{array}$ & $\begin{array}{l}\text { Schyzachyrium } \\
\text { paniculatum }\end{array}$ & pelé Ikaiít (cola de ciervo) & DE \\
\hline Setaria lachnea (Nees) Kunth & Setaria argentina & taasót & NP \\
\hline Sorghastrum pellitum (Hack.) Parodi & Sorghastrum nutans & koñilík, koñillek (lugar donde amarillea) & DE \\
\hline $\begin{array}{l}\text { Sorghastrum setosum } \\
\text { (Griseb.) Hitchc. }\end{array}$ & Sorghastrum agrostoides & koñilík, koñillek (lugar donde amarillea) & DE \\
\hline Steinchisma hians (Elliott) Nash & Panicum milioides & mañík radé (pestaña del suri) & DE \\
\hline Steinchisma laxa (Sw.) Zuloaga & Panicum laxum (?) & penék & NP \\
\hline $\begin{array}{l}\text { Trichloris pluriflora E. } \\
\text { Fourn. f. pluriflora }\end{array}$ & Trichloris pluriflora & uak'apí & NP \\
\hline Triticum aestivum L. & Triticum aestivum & chirígo & PRE \\
\hline Zea mays L.* & Zea mays & nasolék & NP \\
\hline Zea mays var. oryzaea L.* & Zea mays var. oryzaea & nasolék letá-okí (padrecito del maíz) & NP2 \\
\hline
\end{tabular}


G. F. Scarpa y C. N. Rosso - La etnobotánica moqoit inédita de Raúl Martínez Crovetto

\begin{tabular}{|c|c|c|c|}
\hline Especie actual & Especie M.C. (lit.) & Fitónimo moqoit (castellano) & Tipo \\
\hline \multicolumn{4}{|l|}{ POLYGALACEAE } \\
\hline $\begin{array}{l}\text { Asemeia extraaxillaris (Chodat) } \\
\text { J.F.B. Pastore \& J.R. Abbott }\end{array}$ & Polygala extraaxyllaris & nasaló (ha)lók (comida de la perdiz) & DE \\
\hline Polygala duarteana A. St.-Hil. \& Moq. & Polygala duarteana & $\begin{array}{l}\text { nasaló nemaík (amuleto de la perdiz); } \\
\text { nasaló (ha)lók (comida de la perdiz) }\end{array}$ & $\begin{array}{l}\mathrm{DE} \\
\mathrm{DE}\end{array}$ \\
\hline $\begin{array}{l}\text { Polygala molluginifolia } \\
\text { A. St.-Hil. \& Moq. }\end{array}$ & Polygala molluginifolia & $\begin{array}{l}\text { nasaló nemaík (amuleto de la perdiz); } \\
\text { nasaló (ha)lók (comida de la perdiz) }\end{array}$ & $\begin{array}{l}\mathrm{DE} \\
\mathrm{DE}\end{array}$ \\
\hline \multicolumn{4}{|l|}{ POLYGONACEAE } \\
\hline Polygonum acuminatum Kunth & Polygonum acuminatum & tak'ák' lechí (pata de chajá) & DE \\
\hline Polygonum hispidum Kunth & Polygonum hispidum & tak'ák' lechí (pata de chajá) & $\mathrm{DE}$ \\
\hline Polygonum punctatum Elliott & Polygonum punctatum & tak'ák' lechí (pata de chajá) & DE \\
\hline Rumex crispus L. & Rumex crispus & Ioler'ranarát waká (lengua de vaca) & $\mathrm{DE}$ \\
\hline Rumex obtusifolius L. & Rumex obtusifolius & loler'ranarát waká (lengua de vaca) & DE \\
\hline Rumex paraguayensis D. Parodi & Rumex paraguayense & Ioler'ranarát waká (lengua de vaca) & $\mathrm{DE}$ \\
\hline Rumex pulcher L. & Rumex pulcher & Ioler'ranarát waká (lengua de vaca) & $\mathrm{DE}$ \\
\hline \multicolumn{4}{|l|}{ POLYPODIACEAE } \\
\hline $\begin{array}{l}\text { Microgramma vacciniifolia } \\
\text { (Langsd. \& Fisch.) Copel. }\end{array}$ & $\begin{array}{l}\text { Polypodium } \\
\text { vaccciinifolium }\end{array}$ & $\begin{array}{l}\text { k'onasé laañík (soga de la } \\
\text { mujer duende del monte) }\end{array}$ & DE \\
\hline Polypodium sp. & $\begin{array}{l}\text { Polypodium } \\
\text { polypodioides }\end{array}$ & netarguék Ikelá (oreja del itín) & NP2 \\
\hline \multicolumn{4}{|l|}{ PONTEDERIACEAE } \\
\hline Eichhornia crassipes (Mart.) Solms & Eichhornia crassipes & naanók lokó (donde come yacaré) & DE \\
\hline Heteranthera zosterifolia Mart. & Heteranthera zosterifolia & naanók lapotó (poncho del yacaré) & $\mathrm{DE}$ \\
\hline Pontederia cordata L. var. cordata & Pontederia cordata & $\begin{array}{l}\text { naanók lapotó (poncho del yacaré); } \\
\text { naanók lokó (donde come yacaré) }\end{array}$ & $\begin{array}{l}\mathrm{DE} \\
\mathrm{DE}\end{array}$ \\
\hline Pontederia rotundifolia L. f. & Pontederia rotundifolia & $\begin{array}{l}\text { naanók lapotó (poncho del yacaré); } \\
\text { naanók lokó (donde come yacaré) }\end{array}$ & $\begin{array}{l}\mathrm{DE} \\
\mathrm{DE}\end{array}$ \\
\hline \multicolumn{4}{|l|}{ PORTULACACEAE } \\
\hline Portulaca cryptopetala Speg. & Portulaca aff. criptopetala & lo'ió ochisé (gordura o sebo del monte) & DE \\
\hline Portulaca grandiflora Hook. & $\begin{array}{l}\text { Portulaca grandiflora } \\
\text { (M711) }\end{array}$ & togo-olé (rojita) & $\mathrm{DE}$ \\
\hline Portulaca oleracea L. & Portulaca oleracea & lo'ió (gorda, carnosa) & DE \\
\hline \multicolumn{4}{|l|}{ PTERIDACEAE } \\
\hline Adiantopsis dichotoma (Sw.) T. Moore & Adiantopsis dichotoma & layor-r'ái Ikaiít (cola del oso hormiguero) & DE \\
\hline Adiantum raddianum C. Presl. & Adiantum cuneatum & layor-r'ái Ikaiít (cola del oso hormiguero) & $\mathrm{DE}$ \\
\hline \multicolumn{4}{|l|}{ RANUNCULACEAE } \\
\hline $\begin{array}{l}\text { Clematis montevidensis } \\
\text { Spreng. var. montevidensis }\end{array}$ & Clematis hilarii & $\begin{array}{l}\text { nakoló', nokoló'; naiiapëk } \\
\text { lakarebé (barba del diablo) }\end{array}$ & $\begin{array}{l}\text { NP } \\
\text { DE }\end{array}$ \\
\hline \multicolumn{4}{|l|}{ RHAMNACEAE } \\
\hline Ziziphus mistol Griseb. & Ziziphus mistol & nallík & NP \\
\hline \multicolumn{4}{|l|}{ ROSACEAE } \\
\hline Prunus persica (L.) Batsch & Prunus persica & ladájna / ladasna / lodájna & PRE \\
\hline \multicolumn{4}{|l|}{ RUBIACEAE } \\
\hline $\begin{array}{l}\text { Galianthe centranthoides (Cham. } \\
\text { \& Schltdl.) E.L. Cabral }\end{array}$ & Borreria centranthoides & toguiguí / togui ík (colorado por dentro) & $\mathrm{DE}$ \\
\hline $\begin{array}{l}\text { Mitracarpus megapotamicus } \\
\text { (Spreng.) Kuntze }\end{array}$ & Mitracarpus sellowianus & nesor'oná lok (comida del cuis) & DE \\
\hline Richardia brasiliensis Gomes & Richardia brasiliensis & nesor'oná lok (comida del cuis) & DE \\
\hline \multicolumn{4}{|l|}{ RUTACEAE } \\
\hline Citrus sinensis L. Osbeck* & Citrus sinensis & narankík, madankík & PRE \\
\hline Zanthoxylum rhoifolium Lam. & Fagara rhoifolia & k'añík lateé (madre del palo borracho) & NP2 \\
\hline Zanthoxylum sp. & Fagara sp. (M687) & penagarik / penak'-rik (anaranjado) & $\mathrm{DE}$ \\
\hline
\end{tabular}


Bol. Soc. Argent. Bot. 49 (4) 2014

\begin{tabular}{|c|c|c|c|}
\hline Especie actual & Especie M.C. (lit.) & Fitónimo moqoit (castellano) & Tipo \\
\hline Zanthoxylum sp. & Fagara sp. & panrarík (anaranjado) & DE \\
\hline \multicolumn{4}{|l|}{ SALICACEAE } \\
\hline $\begin{array}{l}\text { Salix humboldtiana Willd. } \\
\text { var. humboldtiana }\end{array}$ & Salix chilensis & limak'ñichí lkoík (donde come el picaflor) & DE \\
\hline \multicolumn{4}{|l|}{ SALVINIACEAE } \\
\hline Salvinia auriculata Aubl. & Salvinia auriculata & k'ol & NP \\
\hline Salvinia auriculata Aubl. & Salvinia rotundifolia & k'ol & NP \\
\hline \multicolumn{4}{|l|}{ SANTALACEAE } \\
\hline Acanthosyris falcata Griseb. & Acanthosyris falcata & lar'ái lokoík (donde come la loca) & DE \\
\hline SAPINDACEAE & Sapindaceae (M676) & nowelá & NP \\
\hline $\begin{array}{l}\text { Allophyllus edulis (A. St.-Hil., A. Juss. } \\
\text { \& Cambess.) Hieron. ex Niederl. }\end{array}$ & Allophyllus edulis & k'ochiñí lokoík (donde come la charata) & DE \\
\hline $\begin{array}{l}\text { Allophyllus guaraniticus } \\
\text { (A. St.-Hil.) Radlk. }\end{array}$ & Allophyllus guaraniticus & k'ochiñí lokoík (donde come la charata) & DE \\
\hline Cardiospermum pterocarpum Radlk & $\begin{array}{l}\text { Cardiospermum } \\
\text { pterocarpum }\end{array}$ & nowelá & NP \\
\hline cf. Diplokeleba floribunda N.E. Br. & (“palo piedra”) M678 & chiguisé latak'arík (planta de piedra) & PRE \\
\hline \multicolumn{4}{|l|}{ SAPOTACEAE } \\
\hline $\begin{array}{l}\text { Sideroxylon obtusifolium (Roem. } \\
\text { \& Schult.) T.D. Penn. }\end{array}$ & Bumelia obtusifolia & werakañík, werak r'añík & NP \\
\hline \multicolumn{4}{|l|}{ SCHIZAEACEAE } \\
\hline Aneimia tomentosa (Savigny) Sw. & Aneimia tomentosa & layor-r'ái lkaiít (cola del oso hormiguero) & DE \\
\hline \multicolumn{4}{|l|}{ SCROPHULARIACEAE } \\
\hline Stemodia sp. & Stemodia sp. (M698) & rachipiolé (reventoncito) & DE \\
\hline \multicolumn{4}{|l|}{ SIMAROUBACEAE } \\
\hline Castela coccinea Griseb. & Castela coccinea & tok leí (líquido colorado) & DE \\
\hline Castela tweedii Planch. & Castela sp. (M674) & $\begin{array}{l}\text { shilkáik lokoík (donde come } \\
\text { la iguana o lagartija) }\end{array}$ & $\mathrm{DE}$ \\
\hline \multicolumn{4}{|l|}{ SMILACACEAE } \\
\hline Smilax campestris Griseb. & Smilax campestris & nallín lowé (diente de la tararira) & DE \\
\hline \multicolumn{4}{|l|}{ SOLANACEAE } \\
\hline Capsicum annuum L.* & Capsicum annuum & chemmak'-raík (picante) & DE \\
\hline Capsicum baccatum L. var. baccatum & Capsicum microcarpum & chemmak'-raík (picante) & $\mathrm{DE}$ \\
\hline Capsicum chacoense Hunz. & Capsicum chacöense & chemmak'-raík (picante) & DE \\
\hline Cestrum parqui L'Hér. & Cestrum parqui & piók' latarík (remedio del perro) & $\mathrm{DE}$ \\
\hline Cestrum strigilatum Ruiz \& Pav. & $\begin{array}{l}\text { Cestrum calycinum } \\
\text { (M689) }\end{array}$ & pewé lawer-raík (yuyo que ennegrece) & NP2 \\
\hline Datura ferox $\mathrm{L}$. & Datura ferox & $\begin{array}{l}\text { ronái-olé lateé (madre de los } \\
\text { que pinchan o se adhieren) }\end{array}$ & DE \\
\hline Datura inoxia Mill. & Datura meteloides & $\begin{array}{l}\text { ronái-olé lateé (madre de los } \\
\text { que pinchan o se adhieren) }\end{array}$ & DE \\
\hline Lycium boerhaviaefolium L. f. & Grabowskia duplicata & hoo-wé (sal) & DE \\
\hline Lycium cestroides Schltdl. & Lycium cestroides & k'ochiñí lokoík (donde come la charata) & DE \\
\hline Nicotiana glauca Graham & Nicotiana glauca & neneté latá' (remedio para el grano) & DE \\
\hline Nicotiana longiflora Cav. & Nicotiana longiflora & pewé wer'asar'á (yuyo + ?) & NP2 \\
\hline Nicotiana paa Mart. Crov. & Nicotiana paa & l'paá (su raíz) & $\mathrm{DE}$ \\
\hline Nicotiana tabacum L. & Nicotiana tabacum & naserék / naserík & NP \\
\hline $\begin{array}{l}\text { Petunia axillaris (Lam.) } \\
\text { Britton, Stern \& Poggenb. ssp. } \\
\text { parodii (Steere) Cabrera }\end{array}$ & Petunia axillaris & lak'aorói (dormilona) & $\mathrm{DE}$ \\
\hline Physalis viscosa L. & Physalis viscosa & k'otañí & NP \\
\hline Salpichroa origanifolia (Lam.) Baill. & Salpichroa origanifolia & k'opí & NP \\
\hline
\end{tabular}




\section{G. F. Scarpa y C. N. Rosso - La etnobotánica moqoit inédita de Raúl Martínez Crovetto}

\begin{tabular}{|c|c|c|c|}
\hline Especie actual & Especie M.C. (lit.) & Fitónimo moqoit (castellano) & Tipo \\
\hline Solanum amygdalifolium Steud. & Solanum amygdalifolium & nowelá & NP \\
\hline Solanum angustifidum Bitter & Solanum angustifidum & k'ochiñí lokoík (donde come la charata) & $\mathrm{DE}$ \\
\hline Solanum chacoense Bitter & Solanum chacöense & ioló lokó (comida del pecarí) & DE \\
\hline Solanum commersonii Dunal ex Poir. & Solanum commersonii & ioló lokó (comida del pecarí) & DE \\
\hline Solanum elaeagnifolium Cav. & Solanum eleagnifolium & mañik Ikooté / nkooté (ojo del suri) & DE \\
\hline Solanum sisymbriifolium Lam. & Solanum sisymbriifolium & togué' hâlá (fruta colorada) & DE \\
\hline Solanum sp. & Solanum sp. & lak'aßiar'aík (quebradizo) & $\mathrm{DE}$ \\
\hline Solanum sp. & Solanum sp. (M685) & pewé & NP \\
\hline Solanum sp. & Solanum aff. insidiosum & mañik Ikooté / nkooté (ojo del suri) & DE \\
\hline Vassobia breviflora (Sendtn.) Hunz. & Acnistus breviflorus & piók' latarík (remedio del perro) & DE \\
\hline \multicolumn{4}{|l|}{ TALINACEAE } \\
\hline Talinum fruticosum (L.) Juss. & Talinum racemosum & $\begin{array}{l}\text { kos nemaík (amuleto del } \\
\text { cerdo); pallak' tGáik }\end{array}$ & $\begin{array}{l}\text { DE } \\
\text { NP }\end{array}$ \\
\hline \multicolumn{4}{|l|}{ TYPHACEAE } \\
\hline Typha angustifolia L. & Typha angustifolia & natál, nenowiar'á & $\begin{array}{l}\text { NP; } \\
?\end{array}$ \\
\hline Typha domingensis Pers. & Typha dominguensis & natál, nenowiar'á & $\begin{array}{l}\text { NP; } \\
?\end{array}$ \\
\hline \multicolumn{4}{|l|}{ URTICACEAE } \\
\hline \multirow[t]{2}{*}{ Parietaria debilis G. Forst. } & Parietaria debilis & pok'oík lateé (madre del que quema) & $\mathrm{DE}$ \\
\hline & & k'opi letaá (padre del k’opi) & NP2 \\
\hline Urera baccifera (L.) Gaudich. & Urera baccifera & nanáik lowé (diente de la yarará) & $\mathrm{DE}$ \\
\hline $\begin{array}{l}\text { Urera caracasana (Jacq.) } \\
\text { Gaudich. ex Griseb. }\end{array}$ & Urera caracassana & nanáik lowé (diente de la yarará) & DE \\
\hline Urtica urens L. & Urtica urens & pok'oík (que quema) & DE \\
\hline \multicolumn{4}{|l|}{ VERBENACEAE } \\
\hline $\begin{array}{l}\text { Aloysia gratissima (Gillies } \\
\text { \& Hook. ex Hook.) var. } \\
\text { chacoensis (Moldenke) Botta }\end{array}$ & Aloysia gratissima & k'agueretá lechí (pata del guazuncho) & DE \\
\hline Glandularia peruviana (L.) Small & Glandularia peruviana & pewé togoolé; togoolé (yuyo colorado) & $\begin{array}{l}\text { NP2; } \\
\text { DE }\end{array}$ \\
\hline Lantana balansae Briq & Lantana balansae & koñilalá nemaík (amuleto de la avispa rubita) & $\mathrm{DE}$ \\
\hline $\begin{array}{l}\text { Lippia alba (Mill.) N.E. Br. ex } \\
\text { Britton \& P. Wilson var. alba } \\
\text { VISCACEAE }\end{array}$ & Lippia alba & $\begin{array}{l}\text { ik'olák le’noßiar'ái lateé } \\
\text { (madre de la flor azul) }\end{array}$ & DE \\
\hline Phoradendron argentinum Urb. & Phoradendron pruinosum & k(')ak(')aré Ikaík (cabeza de carancho) & DE \\
\hline Phoradendron bathyoryctum Eichler & Phoradendron hieronymi & k(')ak(')aré Ikaík (cabeza de carancho) & DE \\
\hline $\begin{array}{l}\text { Phoradendron liga (Gillies } \\
\text { ex Hook. \& Arn.) Eichler }\end{array}$ & Phoradendron liga & k(')ak(')aré lkaík (cabeza de carancho) & DE \\
\hline $\begin{array}{l}\text { Phoradendron obtusissimum } \\
\text { (Miq.) Eichler }\end{array}$ & $\begin{array}{l}\text { Phoradendron } \\
\text { acinacifolium }\end{array}$ & k(')ak(')aré lkaík (cabeza de carancho) & DE \\
\hline \multicolumn{4}{|l|}{ VITACEAE } \\
\hline Vitis labrusca L.* & Vitis labrusca & óa-lashí (palo donde se enrosca la uva) & DE \\
\hline \multicolumn{4}{|l|}{ ZYGOPHYLLACEAE } \\
\hline $\begin{array}{l}\text { Porlieria microphylla (Baill.) } \\
\text { Descole, O’Donell \& Lourteig }\end{array}$ & Porlieria microphylla & peter'anr'aík & NP \\
\hline
\end{tabular}


